\title{
Characterizations of intrinsic rectifiability in Heisenberg groups
}

\author{
Pertti Mattila, Raul Serapioni and Francesco Serra Cassano
}

\begin{abstract}
We define rectifiable sets in the Heisenberg groups as countable unions of Lipschitz images of subsets of a Euclidean space, in the case of lowdimensional sets, or as countable unions of subsets of intrinsic $C^{1}$ surfaces, in the case of low-codimensional sets. We characterize both low-dimensional rectifiable sets and low codimensional rectifiable sets with positive lower density, in terms of almost everywhere existence of approximate tangent subgroups or of tangent measures.
\end{abstract}

Mathematics Subject Classification (2010): 28A75 (primary); 28C10 (secondary).

\section{Introduction}

Rectifiable sets are basic concepts of geometric measure theory. They were introduced in the 1920's in the plane by Besicovitch and in 1947 in general dimensions in Euclidean spaces by Federer. A systematic study of rectifiable sets in general metric spaces was made by Ambrosio and Kirchheim in [2] in 2000. However, the definitions they used are not always appropriate in Heisenberg groups, and many other Lie groups, when equipped with their natural Carnot-Carathéodory metric; indeed, often there are only trivial rectifiable sets of measure zero.

A different definition, in Heisenberg groups $\mathbb{H}^{n}$ and more general Carnot groups, has been given in [14], for sets of codimension 1, and in general dimensions in $[17,18]$. Let us sketch the inspiring ideas.

According to Federer's original definition, $k$-dimensional rectifiable sets are contained, up to a negligible set, in countable unions of Lipschitz images of subsets of $\mathbb{R}^{k}$. In Euclidean spaces, it is equivalent to use coverings with countable unions of $k$-dimensional $C^{1}$ sub-manifolds (see [12,31]).

The authors are supported by GALA project of the $6^{\text {th }}$ framework programme of the E.U. P. Mattila is supported by the Academy of Finland, R. Serapioni and F. Serra Cassano are supported by University of Trento and MIUR, Italy.

Received March 2, 2009; accepted in revised form November 2, 2009. 
Also in groups it is possible to follow the pattern of both definitions, provided we have good intrinsic notions of Lipschitz maps and of $C^{1}$ sub-manifolds. Respectively we will define the classes of $\left(k, \mathbb{H}_{L}\right)$-rectifiable sets and of $(k, \mathbb{H})$-rectifiable sets.

Let us begin with the notion of intrinsic $C^{1}$ sub-manifold (see Definition 2.10). If $k$ is an integer, $1 \leq k \leq 2 n$, intrinsic $k$-dimensional $C^{1}$ sub-manifolds of $\mathbb{H}^{n}$ (also called H-regular surfaces) are locally defined, if $1 \leq k \leq n$, as images of continuously differentiable maps $\mathbb{R}^{k} \rightarrow \mathbb{H}^{n}$ and, if $n+1 \leq k \leq 2 n$, as non critical level sets of continuously differentiable functions $\mathbb{H}^{n} \rightarrow \mathbb{\mathbb { R }}^{2 n+\overline{1}-k}$.

Notice that differentiable means always Pansu differentiable (see Definition 2.5). We recall that, if $d$ is the distance, defined in (2.1), equivalent with the CarnotCarathéodory metric, and if $\mathcal{S}^{k}$ is the $k$-dimensional spherical Hausdorff measure in $\mathbb{H}^{n}$, built using the distance $d$, then the Hausdorff dimension of $\mathbb{H}^{n}$ is $2 n+2$ and a (typical) $k$-dimensional $\mathbb{H}$-regular sub-manifold has Hausdorff dimension $k_{m}$, where $k_{m}=k$, if $1 \leq k \leq n$, and $k_{m}=k+1$, if $n+1 \leq k \leq 2 n$.

Then we say that $E \subset \mathbb{H}^{n}$ is $(k, \mathbb{H})$-rectifiable if there is a sequence of $k$ dimensional $\mathbb{H}$-regular surfaces $S_{i}$ such that

$$
\mathcal{S}^{k_{m}}\left(E \backslash \bigcup_{i} S_{i}\right)=0 .
$$

We introduce also the following analogue of Federer's Lipschitz definition. If $k$ is an integer with $1 \leq k \leq 2 n$, we say that $E \subset \mathbb{H}^{n}$ is $\left(k, \mathbb{H}_{L}\right)$-rectifiable if there is a sequence of Lipschitz functions $f_{i}: A_{i} \subset \mathbb{R}^{k} \rightarrow \mathbb{H}^{n}$ such that

$$
\mathcal{S}^{k_{m}}\left(E \backslash \bigcup_{i} f_{i}\left(A_{i}\right)\right)=0 .
$$

This last definition is non trivial only if $1 \leq k \leq n$, because, for $n+1 \leq k$ and $f$ Lipschitz, always $S^{k_{m}}(f(A))=0$ (see [2]), hence we will consider $\left(k, \mathbb{H}_{L}\right)$ rectifiable sets only for $1 \leq k \leq n$.

Given that the above mentioned generalization of Federer's Lipschitz definition is not a good one for high dimensional rectifiable sets in Carnot groups, different related approaches have been proposed in the literature. As an example, Pauls in [28] considered images in $\mathbb{H}^{n}$ of Lipschitz maps defined not on $\mathbb{R}^{k}$ but on subgroups of $\mathbb{H}^{n}$; in [18] a different notion of intrinsic Lipschitz sub-manifold is considered.

We explicitly observe that we do not know if $\left(k, \mathbb{H}_{L}\right)$-rectifiability and $(k, \mathbb{H})$ rectifiability are equivalent if $1 \leq k \leq n$. Clearly they are not if $n+1 \leq k \leq 2 n$.

In this paper we approach the problem of characterizing intrinsic $k$-rectifiable sets in $\mathbb{H}^{n}$ through almost everywhere existence and uniqueness of generalized tangent subgroups or tangent measures.

We prove two main theorems. Suppose that $E \subset \mathbb{H}^{n}$ has locally finite $\mathcal{S}^{k_{m}}$ measure. 
- The first one, Theorem 3.14, deals with the case $1 \leq k \leq n$ and with $\left(k, \mathbb{H}_{L}\right)$ rectifiability. We prove that $E$ is $\left(k, \mathbb{H}_{L}\right)$-rectifiable if and only if it has an approximate tangent subgroup, $\mathcal{S}^{k}$ almost everywhere. The latter means that, for $\mathcal{S}^{k}$ almost all $p \in E$, there exists a homogeneous subgroup $\mathbb{T}_{p}$, of topological and Hausdorff dimension $k$, such that

$$
\lim _{r \rightarrow 0} r^{-k} \mathcal{S}^{k}\left(E \cap B(p, r) \cap\left\{q: d\left(p^{-1} \cdot q, \mathbb{T}_{p}\right)>s d(p, q)\right\}\right)=0
$$

for all $s>0$, where the distance $d$ is defined in (2.1) and is comparable with the Carnot-Carathéodory distance in $\mathbb{H}^{n}$.

- The second main theorem, Theorem 3.15, deals with $n+1 \leq k \leq 2 n$ and $(k, \mathbb{H})$ rectifiability. We prove that $E$ is $(k, \mathbb{H})$-rectifiable if and only if it has, $\mathcal{S}^{k_{m}}$ almost everywhere, an approximate tangent subgroup and, additionally, positive lower density. These two conditions mean that, for $\mathcal{S}^{k_{m}}$ almost all $p \in E$, there exists a homogeneous subgroup $\mathbb{T}_{p}$, of topological dimension $k$ and Hausdorff dimension $k_{m}=k+1$, such that

$$
\lim _{r \rightarrow 0} r^{-k_{m}} \mathcal{S}^{k_{m}}\left(E \cap B(p, r) \cap\left\{q: d\left(p^{-1} \cdot q, \mathbb{T}_{p}\right)>s d(p, q)\right\}\right)=0
$$

for all $s>0$, and

$$
\liminf _{r \rightarrow 0} r^{-k_{m}} \mathcal{S}^{k_{m}}(E \cap B(p, r))>0 .
$$

We do not know if the assumption of positive lower density is necessary.

In both cases, we shall also give other equivalent conditions in terms of weak convergence of blow-ups and existence of tangent measures. One of these, condition (v) of Theorem 3.15 , says that $E$, with positive density as above, is $(k, \mathbb{H})$ rectifiable if and only if for $\mathcal{S}^{k_{m}}$-a.e. $p \in E$ there is a non-trivial Radon measure $\lambda_{p}$ such that every tangent measure at $p$ of the restriction $\mathcal{S}^{k_{m}}\llcorner E$ is a constant multiple of $\lambda_{p}$. More informally, this means that, at all sufficiently small scales around $p, \mathcal{S}^{k_{m}}\left\llcorner E\right.$ looks, suitably magnified, like $\lambda_{p}$. Note that this condition does not refer to any particular kind of subgroups, parametrizations or concepts of differentiability, whence its equivalence to rectifiability in a way indicates that the concept of intrinsic rectifiability we are using is a natural one.

Notice that Ambrosio and Kirchheim's definition in general metric spaces, when specialized to Heisenberg groups, coincides with $\left(k, \mathbb{H}_{L}\right)$-rectifiability. They also have a characterization of rectifiability, under the assumption of positive lower density, in terms of approximate tangent planes. Their approach to the notion of (approximate) tangent space is different from ours. Indeed they imbed their metric space into a Banach space and use the linear structure there. However, eventually, the two notions coincide.

Notice that we use the spherical Hausdorff measure $\mathcal{S}^{m}$ instead of the usual Hausdorff measure $\mathcal{H}^{m}$ because the blow-up Theorem 3.4 is only known for it and 
hence we can get the conditions (ii) and (iv), of both main theorems, only for the spherical Hausdorff measure. As $\mathcal{S}^{m}$ and $\mathcal{H}^{m}$ have the same null-sets, and even $\mathcal{H}^{m}(A) \leq \mathcal{S}^{m}(A) \leq 2^{m} \mathcal{H}^{m}(A)$ for all $A \subset \mathbb{H}^{n}$, the rectifiable sets are the same for both measures and the equivalence of the conditions (i), (iii) and (v) in Theorem 3.14 and 3.15 holds also for $\mathcal{H}^{m}$.

In $[14,15],(2 n, \mathbb{H})$-rectifiable sets were used to establish De Giorgi's structure theory for sets of finite perimeter in step 2 Carnot groups, see also [3] for more general Carnot groups. In Euclidean spaces, rectifiable sets have been fundamental in many aspects of geometric calculus of variations, in particular for minimal surfaces of general dimensions. Problems of existence and regularity of minimal surfaces in Heisenberg groups have recently been considered by many people and it could be expected that rectifiable sets would play a significant role also here. See also, for a different application, the recent [10].

Finally we thank the referee for his careful reading of this paper.

\section{Heisenberg groups}

\subsection{Algebraic and metric structure of $\mathbb{H}^{n}$}

For a general review on Carnot groups, and in particular on Heisenberg groups, we refer to $[8,9,19,32]$ and to [33]. We limit ourselves to fix some notations.

$\mathbb{H}^{n}$ is the n-dimensional Heisenberg group, identified with $\mathbb{R}^{2 n+1}$ through exponential coordinates. A point $p \in \mathbb{H}^{n}$ is denoted $p=\left(p_{1}, \ldots, p_{2 n}, p_{2 n+1}\right)=$ $\left(p^{\prime}, p_{2 n+1}\right)$, with $p^{\prime} \in \mathbb{R}^{2 n}$ and $p_{2 n+1} \in \mathbb{R}$. If $p$ and $q \in \mathbb{H}^{n}$, the group operation is defined as

$$
p \cdot q:=\left(p^{\prime}+q^{\prime}, p_{2 n+1}+q_{2 n+1}-2 \sum_{i=1}^{n}\left(p_{i} q_{i+n}-p_{i+n} q_{i}\right)\right) .
$$

The inverse of $p$ is $p^{-1}:=\left(-p^{\prime},-p_{2 n+1}\right)$ and $e=0$ is the identity of $\mathbb{H}^{n}$.

The centre of $\mathbb{H}^{n}$ is the subgroup $\mathbb{T}:=\left\{p=\left(0, \ldots, 0, p_{2 n+1}\right)\right\}$.

For any $q \in \mathbb{H}^{n}$ and $r>0$, we denote as $\tau_{q}: \mathbb{H}^{n} \rightarrow \mathbb{H}^{n}$ the left translation $p \mapsto q \cdot p=\tau_{q}(p)$ and as $\delta_{r}: \mathbb{H}^{n} \rightarrow \mathbb{H}^{n}$ the dilation

$$
p \mapsto\left(r p^{\prime}, r^{2} p_{2 n+1}\right)=\delta_{r} p
$$

Notice that dilations are also automorphisms of $\mathbb{H}^{n}$.

We denote, for all $p, q \in \mathbb{H}^{n}$,

$$
\|p\|:=d(p, e):=\max \left\{\left\|\left(p_{1}, \cdots, p_{2 n}\right)\right\|_{\mathbb{R}^{2 n}},\left|p_{2 n+1}\right|^{1 / 2}\right\}
$$

and

$$
d(p, q)=d\left(q^{-1} \cdot p, e\right)=\left\|q^{-1} \cdot p\right\|
$$


Then, for all $p, q, z \in \mathbb{H}^{n}$ and for all $r>0$,

$$
d(z \cdot p, z \cdot q)=d(p, q) \quad \text { and } \quad d\left(\delta_{r} p, \delta_{r} q\right)=r d(p, q) .
$$

Finally, $U(p, r)$ and $B(p, r)$ are the open and the closed ball associated with $d$.

The standard basis of the Lie algebra $\mathfrak{h}$ of $\mathbb{H}^{n}$ is given by

$$
X_{i}:=\partial_{i}+2 x_{i+n} \partial_{2 n+1}, \quad Y_{i}:=\partial_{i+n}-2 x_{i} \partial_{2 n+1}, \quad T:=\partial_{2 n+1},
$$

for $i=1, \ldots, n$. The horizontal subspace $\mathfrak{h}_{1}$ is the subspace of $\mathfrak{h}$ spanned by $X_{1}, \ldots, X_{n}$ and $Y_{1}, \ldots, Y_{n}$. Denoting by $\mathfrak{h}_{2}$ the linear span of $T$, the 2-step stratification of $\mathfrak{h}$ is expressed as

$$
\mathfrak{h}=\mathfrak{h}_{1} \oplus \mathfrak{h}_{2} \text {. }
$$

The Lie algebra $\mathfrak{h}$ is endowed with the scalar product $\langle\cdot, \cdot\rangle$, that makes $X_{1}, \ldots, Y_{n}, T$ orthonormal, and by the induced norm $\|\cdot\|$.

The vector fields $X_{1}, \ldots, X_{n}, Y_{1}, \ldots, Y_{n}$ define a vector subbundle of the tangent vector bundle $\mathrm{TH} \mathbb{H}^{n}$, the so called horizontal vector bundle $\mathrm{H} \mathbb{H}^{n}$ - according to the notation of Gromov, (see [19] and [26]). We identify sections $\phi$ of $\mathrm{HH}^{n}$, using coordinates with respect to the moving frame $X_{1}, \ldots, Y_{n}$, with functions $\phi=$ $\left(\phi_{1}, \ldots, \phi_{2 n}\right): \mathbb{H}^{n} \rightarrow \mathbb{R}^{2 n}$. We will also identify sections $\phi: \Omega \subset \mathbb{H}^{n} \rightarrow \mathrm{H} \mathbb{H}^{n}$ with the map $\phi: \Omega \rightarrow \mathfrak{h}_{1}$ defined as

$$
\phi(p)=\sum_{j=1}^{n} \phi_{j}(p) X_{j}+\phi_{n+j}(p) Y_{j} .
$$

We denote by $\pi: \mathbb{H}^{n} \rightarrow \mathfrak{h}_{1}$ the map defined as

$$
\pi(p):=\sum_{j=1}^{n}\left(p_{j} X_{j}+p_{n+j} Y_{j}\right) .
$$

Remark 2.1. Because the topologies defined by $d$ and by the Euclidean distance coincide, the topological dimension of $\mathbb{H}^{n}$ is $2 n+1$. Moreover $d$ is bilipschitz equivalent to the Carnot-Carathéodory metric $d_{c}$ induced by the horizontal subbundle $H \mathbb{H}^{n}$ (see $\left.[19,26]\right)$.

The $(2 n+1)$-dimensional Lebesgue measure $\mathcal{L}^{2 n+1}$ on $\mathbb{H}^{n} \equiv \mathbb{R}^{2 n+1}$ is left (and right) invariant and it is a Haar measure of the group.

For $m \geq 0$, we denote by $\mathcal{S}^{m}$ the $m$-dimensional spherical Hausdorff measure, obtained from the distance $d$ following Carathédory's construction as in [12, Section 2.10.2].

For $A \subset \mathbb{H}^{n}$ and $\delta>0, \mathcal{S}^{m}(A)=\lim _{\delta \rightarrow 0} \mathcal{S}_{\delta}^{m}(A)$, where

$$
\mathcal{S}_{\delta}^{m}(A)=\inf \left\{\sum_{i} \alpha_{m} r_{i}^{m}: A \subset \bigcup_{i} B\left(p_{i}, r_{i}\right), r_{i} \leq \delta\right\} .
$$


We have to be precise about the constants $\alpha_{m}$ appearing in this definition. We will define $\alpha_{m}$ only for $m$ integer, $0 \leq m \leq 2 n+2$ and $m \neq n+1$. Indeed explicit computations will be carried out only in these cases, because, as observed in the introduction, $k$-dimensional $C^{1}$ (or Lipschitz) submanifolds have intrinsic Hausdorff dimension $k$ if $1 \leq k \leq n$ and Hausdorff dimension $k+1$ if $n+1 \leq k \leq$ $2 n+1$. define

Hence, if $\omega_{m}$ is the $\mathcal{L}^{m}$ measure of the unit Euclidean ball $B_{m \text {, euc }}$ in $\mathbb{R}^{m}$, we

$$
\alpha_{m}:= \begin{cases}\omega_{m} & \text { if } 1 \leq m \leq n, \\ 2 \omega_{m-2} & \text { if } n+2 \leq m \leq 2 n+2 .\end{cases}
$$

With this choice, the $\mathcal{S}^{m}$ measure restricted to homogeneous subgroups of $\mathcal{G}\left(\mathbb{H}^{n}\right)$ (see Definition 2.16) coincides with the appropriate Lebesgue measure. The precise statement is in Proposition 2.20.

Translation invariance and homogeneity under dilations of Hausdorff measures follow as usual. For all $A \subseteq \mathbb{H}^{n}$, for all $p \in \mathbb{H}^{n}, r \in(0, \infty)$ and $1 \leq m \leq 2 n+2$

$$
\mathcal{S}^{m}\left(\tau_{p} A\right)=\mathcal{S}^{m}(A), \quad \mathcal{S}^{m}\left(\delta_{r}(A)\right)=r^{m} \mathcal{S}^{m}(A) .
$$

Finally we denote by $\mathcal{H}_{\text {euc }}^{m}$ the $m$-dimensional Hausdorff measure related to the Euclidean distance in $\mathbb{R}^{2 n+1}$.

\subsection{Function spaces}

If $\Omega$ is an open subset of $\mathbb{H}^{n} \equiv \mathbb{R}^{2 n+1}$ and $k \geq 0$ is a non negative integer, $\mathbf{C}^{k}(\Omega)$ indicates the usual space of real valued functions which are $k$ times continuously differentiable in the Euclidean sense. We denote by $\mathbf{C}^{k}\left(\Omega, H \mathbb{H}^{n}\right)$ the set of all $C^{k}$-sections of $\mathrm{HH}^{n}$ where the $C^{k}$ regularity is understood as regularity between smooth manifolds.

Definition 2.2. If $\Omega$ is an open subset of $\mathbb{H}^{n}$ and $f \in \mathbf{C}^{1}(\Omega)$ we define the horizontal gradient of $f$ as

$$
\nabla_{H} f:=\left(X_{1} f, \ldots, X_{n} f, Y_{1} f, \ldots, Y_{n} f\right) .
$$

Alternatively $\nabla_{H} f$ can be defined as the section of $\mathrm{HH}^{n}$

$$
\nabla_{H} f:=\sum_{j=1}^{n}\left(X_{j} f\right) X_{j}+\left(Y_{j} f\right) Y_{j}
$$

whose canonical coordinates are $\left(X_{1} f, \ldots, X_{n} f, Y_{1} f, \ldots, Y_{n} f\right)$.

Definition 2.3. We say that $f: \mathbb{H}^{n} \rightarrow \mathbb{R}$ is differentiable along $X_{j}$ (or along $Y_{j}$ ) at $p_{0}$ if the map $r \mapsto f\left(p_{0} \cdot \delta_{r} e_{j}\right)$ (respectively: $r \mapsto f\left(p_{0} \cdot \delta_{r} e_{j+n}\right)$ ) is differentiable at $r=0$. Here $e_{k}$ is the $k$-th vector of the canonical basis of $\mathbb{R}^{2 n+1}$. 
Clearly, if $f \in \mathbf{C}^{1}(\Omega)$ then $f$ is differentiable along $X_{j}$ and $Y_{j}$, for $j=$ $1, \ldots, n$, at all points of $\Omega$ and

$$
\left.\frac{\mathrm{d} f}{\mathrm{~d} r}\left(p_{0} \cdot \delta_{r} e_{j}\right)\right|_{r=0}=X_{j} f\left(p_{0}\right),\left.\quad \frac{\mathrm{d} f}{\mathrm{~d} r}\left(p_{0} \cdot \delta_{r} e_{j+n}\right)\right|_{r=0}=Y_{j} f\left(p_{0}\right)
$$

for all $p_{0} \in \Omega$. Hence, if $f$ is differentiable along $X_{j}$ 's and $Y_{j}$ 's at $p_{0}$ and we define the horizontal gradient to be

$$
\nabla_{H} f\left(p_{0}\right):=\sum_{j=1}^{n} X_{j} f\left(p_{0}\right) X_{j}+\sum_{j=1}^{n} Y_{j} f\left(p_{0}\right) Y_{j}
$$

then this definition naturally extends the one given in (2.5).

Definition 2.4. If $\mathcal{U} \subset \mathbb{H}^{n}$, we denote by $\mathbf{C}_{H}^{1}(\mathcal{U})$ the set of continuous real valued functions in $\mathcal{U}$ such that $\nabla_{H} f$ exists and is continuous in $\mathcal{U} ;\left[\mathbf{C}_{H}^{1}(\mathcal{U})\right]^{k}$ is the set of $k$-tuples $f=\left(f_{1}, \cdots, f_{k}\right)$ such that each $f_{i} \in \mathbf{C}_{H}^{1}(\mathcal{U})$ for $1 \leq i \leq k$.

Notice that $\mathbf{C}^{1}(\Omega) \subset \mathbf{C}_{H}^{1}(\Omega)$, and the inclusion is strict (see [14, Remark 5.9]). The following definition of differentiability, for functions acting between Carnot groups, was given by Pansu in [27].

Definition 2.5. Let $\left(\mathbb{G}_{1}, d_{1}\right)$ and $\left(\mathbb{G}_{2}, d_{2}\right)$ be Carnot groups and $\mathcal{A} \subset \mathbb{G}_{1}$. A function $f: \mathcal{A} \rightarrow \mathbb{G}_{2}$ is Pansu differentiable in $g \in \mathcal{A}$ if there is a homogeneous homomorphism $L_{g}: \mathbb{G}_{1} \rightarrow \mathbb{G}_{2}$ such that

$$
\frac{d_{2}\left(f(g)^{-1} \cdot f\left(g^{\prime}\right), L_{g}\left(g^{-1} \cdot g^{\prime}\right)\right)}{d_{1}\left(g, g^{\prime}\right)} \rightarrow 0, \quad \text { as } d_{1}\left(g, g^{\prime}\right) \rightarrow 0, g^{\prime} \in \mathcal{A} .
$$

The homogeneous homomorphism $L_{g}$ is denoted $d_{H} f_{g}$ and is called the Pansu differential of $f$ in $g$.

Pansu obtained, in [27], a Rademacher type theorem for Lipschitz functions acting between Carnot groups proving that they are almost everywhere differentiable, in the sense of Definition 2.5. We shall use the following case of Pansu's theorem, (see [21, Theorem 3.4.11]).

Theorem 2.6. Let $A \subset \mathbb{R}^{m}$ be an $\mathcal{L}^{m}$ measurable set and $f: \mathcal{A} \rightarrow \mathbb{H}^{n}$ be a Lipschitz map. Then $f$ is Pansu differentiable $\mathcal{L}^{m}$-a.e. in $\mathcal{A}$.

The functions of $\mathbf{C}_{H}^{1}(\Omega)$ are differentiable in Pansu's sense (see [27] and [14]) and the Pansu differential $d_{H} f$ is represented by the horizontal gradient $\nabla_{H} f$.

Proposition 2.7. With the notations of Definition 2.3, $f \in \mathbf{C}_{H}^{1}(\Omega)$ if and only if its distributional derivatives $X_{i} f, Y_{i} f(i=1, \ldots, n)$ are continuous in $\Omega$.

Moreover, if $f \in \mathbf{C}_{H}^{1}(\Omega)$, then for all $p, p_{0} \in \Omega$

$$
f(p)=f\left(p_{0}\right)+d_{H} f_{p_{0}}\left(p_{0}^{-1} \cdot p\right)+o\left(d\left(p, p_{0}\right)\right), \quad \text { as } p \rightarrow p_{0} .
$$


We mention also the following 'converse' result to Theorem 2.6.

Proposition 2.8. Let $1 \leq d \leq n$. If $f: \mathcal{A} \subset \mathbb{R}^{d} \rightarrow \mathbb{H}^{n}$ is Pansu differentiable in $x_{0}$ then $f$ is also Euclidean differentiable in $x_{0}$ and

$$
f\left(x_{0}\right) \cdot d_{H} f_{x_{0}}\left(\mathbb{R}^{d}\right)=f\left(x_{0}\right)+d f_{x_{0}}\left(\mathbb{R}^{d}\right) .
$$

Proof. By the contact property of H-linear maps (see [21] or [17]) we know that $L:=d_{H} f_{x_{0}}$ is a classical linear map from $\mathbb{R}^{d}$ to $\mathbb{R}^{2 n+1}$ and $L(v)=(A v, 0)$ where $A$ is a suitable $2 n \times d$ matrix. Then, for the first $2 n$ components $f_{1}, \ldots, f_{2 n}$ of $f$, Pansu differentiability coincides with Euclidean differentiability so that

$$
A=\left[\begin{array}{c}
\nabla f_{1}\left(x_{0}\right) \\
\vdots \\
\nabla f_{2 n}\left(x_{0}\right)
\end{array}\right]
$$

while a not difficult computation yields

$$
\nabla f_{2 n+1}\left(x_{0}\right)=2 \sum_{j=1}^{n}\left(f_{j+n}\left(x_{0}\right) \nabla f_{j}\left(x_{0}\right)-f_{j}\left(x_{0}\right) \nabla f_{j+n}\left(x_{0}\right)\right) .
$$

It follows that, for all $v \in \mathbb{R}^{d}$,

$$
f\left(x_{0}\right) \cdot d_{H} f_{x_{0}}(v)=f\left(x_{0}\right)+d f_{x_{0}}(v)
$$

and the proof is completed.

We conclude this section mentioning the following Whitney's extension theorem (see [14]).

Theorem 2.9. Let $F \subset \mathbb{H}^{n}$ be a closed set, $f: F \rightarrow \mathbb{R}, k: F \rightarrow H \mathbb{H}^{n}$ be continuous functions. Define

$$
R\left(p^{\prime}, p\right):=\frac{f\left(p^{\prime}\right)-f(p)-\left\langle k(p), \pi\left(p^{-1} \cdot p^{\prime}\right)\right\rangle}{d\left(p, p^{\prime}\right)},
$$

and, if $K \subset F$ is a compact set,

$$
\rho_{K}(\delta):=\sup \left\{\left|R\left(p^{\prime}, p\right)\right|: \quad p, p^{\prime} \in K, 0<d\left(p, p^{\prime}\right)<\delta\right\} .
$$

If $\rho_{K}(\delta) \rightarrow 0$ as $\delta \rightarrow 0$ for every compact set $K \subset F$, then there exists $\tilde{f}: \mathbb{H}^{n} \rightarrow$ $\mathbb{R}, \tilde{f} \in \mathbf{C}_{H}^{1}\left(\mathbb{H}^{n}\right)$, such that

$$
\tilde{f}_{\mid F}=f \quad \text { and } \quad \nabla_{H} \tilde{f}_{\mid F}=k .
$$

\section{3. $\mathbb{H}$-regular surfaces}

$\mathbb{H}$-regular surfaces are the intrinsic $C^{1}$ embedded submanifolds in $\mathbb{H}^{n}$. A systematic study of $\mathbb{H}$-regular surfaces has been recently carried out in $[4,5,11,17]$. Let us begin with the definition. 
Definition 2.10. $S \subset \mathbb{H}^{n}$ is a d-dimensional $\mathbb{H}$-regular surface when the following is true

(i) for $1 \leq d \leq n$

for any $p \in S$ there are open sets $\mathcal{U} \subset \mathbb{H}^{n}, \mathcal{V} \subset \mathbb{R}^{d}$ and a function $\varphi: \mathcal{V} \rightarrow \mathcal{U}$ such that $p \in \mathcal{U}, \varphi$ is injective, and continuously Pansu differentiable with $d_{H} \varphi_{p}$ injective, and

$$
S \cap \mathcal{U}=\varphi(\mathcal{V})
$$

(ii) for $n+1 \leq d \leq 2 n$

for any $p \in S$ there are an open set $\mathcal{U} \subset \mathbb{H}^{n}$ and a function $f: \mathcal{U} \rightarrow \mathbb{R}^{2 n+1-d}$ such that $p \in \mathcal{U}, f \in\left[\mathbf{C}_{\mathbf{H}}^{\mathbf{1}}(\mathcal{U})\right]^{2 n+1-d}$, with $d_{H} f_{q}$ surjective for every $q \in \mathcal{U}$ and

$$
S \cap \mathcal{U}=\{q \in \mathcal{U}: f(q)=0\} .
$$

In this second case, sometimes we speak of $(2 n+1-d)$-codimensional $\mathbb{H}$-regular surfaces.

These notions of $\mathbb{H}$-regular submanifolds are different from the corresponding Euclidean ones and from each other. Indeed $d$-dimensional $\mathbb{H}$-regular submanifolds, $1 \leq d \leq n$, are a subclass of $d$-dimensional Euclidean $C^{1}$ submanifolds of $\mathbb{R}^{2 n+1}$ (see [17]). On the contrary, $k$-codimensional $H$-regular submanifolds, $1 \leq k \leq n$, can be very irregular objects from a Euclidean point of view. An example of a 1-codimensional $H$-regular surface in $\mathbb{H}^{1} \equiv \mathbb{R}^{3}$, with fractional Euclidean dimension equal to 2.5 , is provided in [20]. On the other side, the horizontal plane $\left\{p: p_{2 n+1}=0\right\}=\exp \mathfrak{h}_{1}$ is Euclidean regular but not intrinsic regular at the origin. Nevertheless, $\mathbb{H}$-regular surfaces share several properties with the Euclidean regular ones. To see this let us first introduce the notion of intrinsic tangent subgroup to an $\mathbb{H}$-regular surface.

Definition 2.11. Let $S$ be a $d$-dimensional $\mathbb{H}$-regular surface in $\mathbb{H}^{n}$ and $\varphi$ and $f$ as in Definition 2.10. The tangent group to $S$ in $p_{0} \in S$, denoted as $T_{\mathbb{H}} S\left(p_{0}\right)$, is the homogeneous subgroup of $\mathbb{H}^{n}$ defined,

(i) for $1 \leq d \leq n$, as

$$
T_{\mathbb{H}} S\left(p_{0}\right):=\left\{d_{H} \varphi_{x_{0}}(x): x \in \mathbb{R}^{d}\right\}, \quad \text { where } \varphi\left(x_{0}\right)=p_{0} .
$$

(ii) for $n+1 \leq d \leq 2 n$, as

$$
T_{\mathbb{H}} S\left(p_{0}\right):=\left\{p \in \mathbb{H}^{n}: d_{H} f_{p_{0}}(p)=0\right\} .
$$

Remark 2.12. If $1 \leq d \leq n$ and if $S$ is a $\mathbb{H}$-regular $d$-dimensional surface, then, for all $p \in S, T_{\mathbb{H}} S(p)$ is a $d$-dimensional subgroup contained in $H \mathbb{H}^{n}$. Moreover the Euclidean tangent plane to $S$ in $p$ coincides with $T_{\mathbb{H}} S(p)$ (see [17, Theorem 3.5]). If $n+1 \leq d \leq 2 n$ and if $S$ is an $\mathbb{H}$-regular $d$-surface, then $T_{\mathbb{H}} S(p)$ is a $d$-dimensional subgroup containing the centre $\mathbb{T}$ of $\mathbb{H}^{n}$ (see [17]). Finally, it follows, from the very Definition 2.10, that $S$ is a $k$-codimensional $\mathbb{H}$-regular surface, $1 \leq k<n$, if and only if $S$ is, locally, the intersection of $k 1$-codimensional $\mathbb{H}$-regular hypersurfaces, with linearly independent normal vectors. 


\subsection{The intrinsic Grassmannian}

A subgroup $\mathbb{G}$ of $\mathbb{H}^{n}$ is a homogeneous subgroup if

$$
\delta_{r} g \in \mathbb{G},
$$

for all $g \in \mathbb{G}$ and for all $r>0$. Notice that, through the identification of $\mathbb{H}^{n}$ with $\mathbb{R}^{2 n+1}$, homogeneous subgroups of $\mathbb{H}^{n}$ are vector subspaces of $\mathbb{R}^{2 n+1}$. Homogeneous subgroups either are contained in the horizontal plane $\exp \mathfrak{h}_{1}$, and are then called horizontal, or they contain the centre $\mathbb{T}$ of $\mathbb{H}^{n}$, and are called vertical. Horizontal subgroups are commutative while vertical subgroups are non-commutative normal subgroups of $\mathbb{H}^{n}$.

We use the symbol $\operatorname{dim} \mathbb{G}$ to indicate the 'linear' dimension of the subgroup $\mathbb{G}$, i.e. the dimension of its Lie algebra, and the symbol $\operatorname{dim}_{\mathbb{H}} \mathbb{G}$ for its Hausdorff dimension, with respect to the metric $d$, defined in (2.1). It follows from a general result of Mitchell (see [25]), and also from direct verification in this case, that $\operatorname{dim}_{\mathbb{H}} \mathbb{V}=\operatorname{dim} \mathbb{V}$, if $\mathbb{V}$ is a horizontal subgroup, and that $\operatorname{dim}_{\mathbb{H}} \mathbb{W}=\operatorname{dim} \mathbb{W}+1$, if $\mathbb{W}$ is a vertical subgroup.

In the following we will refer to homogeneous subgroups $\mathbb{G}$ of linear dimension $d$ as $d$-subgroups and we will denote as $d_{m}$ the metric dimension of $\mathbb{G}$.

Definition 2.13. Two homogeneous subgroups $\mathbb{V}$ and $\mathbb{W}$ of $\mathbb{H}^{n}$ are complementary subgroups in $\mathbb{H}^{n}$, if $\mathbb{W} \cap \mathbb{V}=\{e\}$ and if, for all $p \in \mathbb{H}^{n}$, there are $w \in \mathbb{W}$ and $v \in \mathbb{V}$ such that $p=w \cdot v$. If $\mathbb{W}$ and $\mathbb{V}$ are complementary subgroups, we say that $\mathbb{H}^{n}$ is the product of $\mathbb{W}$ and $\mathbb{V}$ and we write

$$
\mathbb{H}^{n}=\mathbb{W} \cdot \mathbb{V},
$$

Remark 2.14. In $\mathbb{H}^{n}$ one of two complementary subgroups, is always a normal subgroup and the other one a horizontal subgroup. Hence the product $\mathbb{H}^{n}=\mathbb{W} \cdot \mathbb{V}$ in Definition 2.13, is always a semidirect product (see Proposition 2.17). We will use the symbol $\mathbb{W}$ for normal subgroups and the symbol $\mathbb{V}$ for the horizontal ones.

Proposition 2.15. If $\mathbb{H}^{n}=\mathbb{W} \cdot \mathbb{V}$ is as above, with $\mathbb{V}$ horizontal and $\mathbb{W}$ vertical, each $p \in \mathbb{H}^{n}$ has unique components $p_{\mathbb{W}} \in \mathbb{W}, p_{\mathbb{V}} \in \mathbb{V}$, such that

$$
p=p_{\mathbb{W}} \cdot p_{\mathbb{V}}
$$

For all $p, q \in \mathbb{H}^{n}$ and $\lambda>0, p_{\mathbb{W}}$ and $p_{\mathbb{V}}$ depend continuously on $p$ and,

$$
\begin{aligned}
& \left(p^{-1}\right)_{\mathbb{W}}=\left(p_{\mathbb{V}}\right)^{-1} \cdot\left(p_{\mathbb{W}}\right)^{-1} \cdot p_{\mathbb{V}}, \quad\left(p^{-1}\right)_{\mathbb{V}}=\left(p_{\mathbb{V}}\right)^{-1} \\
& \left(\delta_{\lambda} p\right)_{\mathbb{W}}=\delta_{\lambda} p_{\mathbb{W}}, \quad(p \cdot q)_{\mathbb{W}}=p_{\mathbb{W}} \cdot p_{\mathbb{V}} \cdot q_{\mathbb{W}} \cdot p_{\mathbb{V}}^{-1} \\
& \left(\delta_{\lambda} p\right)_{\mathbb{V}}=\delta_{\lambda} p_{\mathbb{V}}, \quad(p \cdot q)_{\mathbb{V}}=p_{\mathbb{V}} \cdot q_{\mathbb{V}},
\end{aligned}
$$

hence, in particular, the map $p \mapsto p_{\mathbb{V}}$, from $\mathbb{H}^{n}$ to $\mathbb{V}$, is a homogeneous homomorphism. 
There is a constant $c=c(\mathbb{W}, \mathbb{V})>0$ such that, for all $p \in \mathbb{H}^{n}$,

$$
\begin{gathered}
c\left(\left\|p_{\mathbb{W}}\right\|+\left\|p_{\mathbb{V}}\right\|\right) \leq\|p\| \leq\left\|p_{\mathbb{W}}\right\|+\left\|p_{\mathbb{V}}\right\|, \\
c\left(\left\|p_{\mathbb{V}}^{-1} \cdot p_{\mathbb{W}} \cdot p_{\mathbb{V}}\right\|+\left\|p_{\mathbb{V}}\right\|\right) \leq\|p\| \leq\left\|p_{\mathbb{V}}^{-1} \cdot p_{\mathbb{W}} \cdot p_{\mathbb{V}}\right\|+\left\|p_{\mathbb{V}}\right\| .
\end{gathered}
$$

and consequently

$$
\begin{aligned}
c\left\|p_{\mathbb{V}}\right\| & \leq d(p, \mathbb{W}) \leq\left\|p_{\mathbb{V}}\right\|, \\
c\left\|p_{\mathbb{V}}^{-1} \cdot p_{\mathbb{W}} \cdot p_{\mathbb{V}}\right\| & \leq d(p, \mathbb{V}) \leq\left\|p_{\mathbb{V}}^{-1} \cdot p_{\mathbb{W}} \cdot p_{\mathbb{V}}\right\| .
\end{aligned}
$$

Proof. Uniqueness, continuity, (2.8) and (2.9) are trivial. Then (2.10) follows from triangular inequality and by a compactness argument, observing also that $p=p_{\mathbb{W}}$. $p_{\mathbb{V}}=p_{\mathbb{V}} \cdot\left(p_{\mathbb{V}}\right)^{-1} \cdot p_{\mathbb{W}} \cdot p_{\mathbb{V}}$.

Because

$$
d(p, \mathbb{W})=\inf \{d(p, w): w \in \mathbb{W}\}=\inf \left\{\left\|w^{-1} \cdot p\right\|: w \in \mathbb{W}\right\}
$$

we get, from (2.10),

$$
d(p, \mathbb{W})=\inf \left\{\left\|w^{-1} \cdot p_{\mathbb{W}} \cdot p_{\mathbb{V}}\right\|: w \in \mathbb{W}\right\} \leq\left\|p_{\mathbb{V}}\right\|
$$

and, on the other side,

$$
\left\|w^{-1} \cdot p_{\mathbb{W}} \cdot p_{\mathbb{V}}\right\| \geq c\left(\left\|w^{-1} \cdot p_{\mathbb{W}}\right\|+\left\|p_{\mathbb{V}}\right\|\right) \geq c\left\|p_{\mathbb{V}}\right\|
$$

that gives the first one of (2.11).

Once more from (2.10),

$$
\begin{aligned}
& d(p, \mathbb{V})=\inf \left\{\left\|p^{-1} \cdot v\right\|: v \in \mathbb{V}\right\} \\
& =\inf \left\{\left\|\left(p_{\mathbb{V}}\right)^{-1} \cdot\left(p_{\mathbb{W}}\right)^{-1} \cdot p_{\mathbb{V}} \cdot\left(p_{\mathbb{V}}\right)^{-1} \cdot v\right\|: v \in \mathbb{V}\right\} \\
& \geq c \inf \left\{\left\|\left(p_{\mathbb{V}}\right)^{-1} \cdot\left(p_{\mathbb{W}}\right)^{-1} \cdot p_{\mathbb{V}}\right\|+\left\|\left(p_{\mathbb{V}}\right)^{-1} \cdot v\right\|: v \in \mathbb{V}\right\} \\
& =c\left\|p_{\mathbb{V}}^{-1} \cdot\left(p_{\mathbb{W}}\right)^{-1} \cdot p_{\mathbb{V}}\right\| \\
& =c\left\|p_{\mathbb{V}}^{-1} \cdot p_{\mathbb{W}} \cdot p_{\mathbb{V}}\right\| \text {. }
\end{aligned}
$$

The estimate from above is obtained in the same way.

Definition 2.16. Let $d$ be a non negative integer. We denote by $\mathcal{H}\left(\mathbb{H}^{n}, d, d_{m}\right)$ the set of all $d$-subgroups of $\mathbb{H}^{n}$ with metric dimension $d_{m}$ and by $\mathcal{H}\left(\mathbb{H}^{n}\right)$ the family of all homogeneous subgroups. A $d$-subgroup $\mathbb{G}$ belongs to the intrinsic Grassmannian of the $d$-subgroups $\mathcal{G}\left(\mathbb{H}^{n}, d\right)$, if there is a $(2 n+1-d)$-subgroup $\mathbb{H}$ such that $\mathbb{H}^{n}=\mathbb{G} \cdot \mathbb{H}$, that is

$$
\mathcal{G}\left(\mathbb{H}^{n}, d\right):=\left\{\mathbb{G}: \begin{array}{l}
\mathbb{G} \text { is a } d \text {-subgroup and } \mathbb{H}^{n}=\mathbb{G} \cdot \mathbb{H} \\
\text { for a }(2 n+1-d) \text {-subgroup } \mathbb{H}
\end{array}\right\} .
$$


The intrinsic Grassmannian is defined as

$$
\mathcal{G}\left(\mathbb{H}^{n}\right)=\bigcup_{d=0}^{2 n+1} \mathcal{G}\left(\mathbb{H}^{n}, d\right)
$$

Proposition 2.17. The trivial subgroups $\{e\}$ and $\mathbb{H}^{n}$ are the unique elements of, respectively, $\mathcal{G}\left(\mathbb{H}^{n}, 0\right)$ and $\mathcal{G}\left(\mathbb{H}^{n}, 2 n+1\right)$. Moreover,

(i) for $1 \leq d \leq n, \mathcal{G}\left(\mathbb{H}^{n}, d\right)=\mathcal{H}\left(\mathbb{H}^{n}, d, d\right)$, the set of all horizontal homogeneous d-subgroups;

(ii) for $n+1 \leq d \leq 2 n, \mathcal{G}\left(\mathbb{H}^{n}, d\right)=\mathcal{H}\left(\mathbb{H}^{n}, d, d+1\right)$ coincides with the set of all vertical homogeneous $d$-subgroups.

On the contrary, a vertical subgroup $\mathbb{W}$ with $1 \leq \operatorname{dim} \mathbb{W} \leq n$ is not in $\mathcal{G}\left(\mathbb{H}^{n}\right)$. In particular, the 1-subgroup $\mathbb{T}$ is not in $\mathcal{G}\left(\mathbb{H}^{n}\right)$.

Proof. In all the possible splittings of $\mathbb{H}^{n}$ as semidirect product of two subgroups, $\mathbb{H}^{n}=\mathbb{W} \cdot \mathbb{V}$, one of the two subgroups, say $\mathbb{V}$, is horizontal and the other one, $\mathbb{W}$, contains $\mathbb{T}$. In terms of Lie algebras, setting $\mathfrak{v}$ and $\mathfrak{w}$ the Lie algebras of $\mathbb{V}$ and $\mathbb{W}$, we have that $\mathfrak{h}=\mathfrak{v} \oplus \mathfrak{w}, \mathfrak{v}$ and $\mathfrak{w}$ are subalgebras of $\mathfrak{h}$ and, $\mathbb{V}$ being horizontal, $\mathfrak{v}$ is commutative.

Hence $0 \leq \operatorname{dim} \mathbb{V} \leq n$ because in $\mathfrak{h}$ there are at most $n$ linearly independent, commuting horizontal vector fields. Consequently, $n<\operatorname{dim} \mathbb{W}=2 n+1-\operatorname{dim} \mathbb{V}<$ $2 n+1$. This shows that vertical subgroups of linear dimension not exceeding $n$ can never be a factor of a splitting of $\mathbb{H}^{n}$.

If $L$ is any vector subspace of $\mathfrak{h}_{1}$ then $\operatorname{span}\{L, T\}$ is a subalgebra of $\mathfrak{h}$ and $\exp \{\operatorname{span}\{L, T\}\}$ is a vertical subgroup of $\mathbb{H}^{n}$. It follows that all the horizontal subgroups are in $\mathcal{G}\left(\mathbb{H}^{n}\right)$. Indeed, given a horizontal subgroup $\mathbb{V}$, its Lie algebra $\mathfrak{v}$ is a vector subspace of $\mathfrak{h}_{1}$. Let $L$ be any complementary vector subspace of $\mathfrak{v}$ in $\mathfrak{h}_{1}$ and define $\mathbb{W}:=\exp \{\operatorname{span}\{L, T\}\}$. Then $\mathbb{H}^{n}$ is the semidirect product of $\mathbb{V}$ and $\mathbb{W}$, so that $\mathbb{V} \in \mathcal{G}\left(\mathbb{H}^{n}\right)$.

On the other side, if $\mathbb{W}$ is a vertical subgroup of dimension larger than $n$ it is proved in Lemma 3.26 of [17] that a complementary horizontal subgroup $\mathbb{V}$ exists such that $\mathbb{H}^{n}$ is the direct product of $\mathbb{W}$ and $\mathbb{V}$.

Remark 2.18. $\mathcal{G}\left(\mathbb{H}^{n}\right)$ and $\mathcal{H}\left(\mathbb{H}^{n}\right)$ are, in a natural way, subsets of the Euclidean Grassmannian $\mathcal{G}\left(\mathbb{R}^{2 n+1}\right)$ and are endowed with the same topology. Moreover notice that $\mathcal{G}\left(\mathbb{H}^{n}, d\right)$ and $\mathcal{H}\left(\mathbb{H}^{n}, d, d_{m}\right)$ are compact metric spaces with respect to the distance

$$
\rho\left(\mathbb{G}_{1}, \mathbb{G}_{2}\right):=d_{\text {Haus }}\left(\mathbb{G}_{1} \cap B(e, 1), \mathbb{G}_{2} \cap B(e, 1)\right),
$$

where $d_{\text {Haus }}$ is the Hausdorff distance induced by the distance $d$.

Remark 2.19. If $S$ is a $d$-dimensional $\mathbb{H}$-regular surface and $p \in S$ then

$$
T_{\mathbb{H}} S(p) \in \mathcal{G}\left(\mathbb{H}^{n}, d\right)
$$


Proposition 2.20. Let $\mathbb{G} \in \mathcal{H}\left(\mathbb{H}^{n}, d, d_{m}\right)$. Then $\mathcal{H}_{\text {euc }}^{d}\llcorner\mathbb{G}$, is a left Haar measure of $\mathbb{G}$ and

$$
\mathcal{H}_{\text {euc }}^{d}\left\llcorner\mathbb{G}=\mathcal{S}^{d_{m}}\llcorner\mathbb{G}\right.
$$

where

$$
\begin{array}{ll}
d_{m}=d & \text { if } \mathbb{G} \text { is a horizontal subgroup, } \\
d_{m}=d+1 & \text { if } \mathbb{G} \text { is a vertical subgroup. }
\end{array}
$$

In particular, if $\mathbb{G} \in \mathcal{G}\left(\mathbb{H}^{n}, d\right)$ then $\operatorname{dim}_{\mathbb{H}} \mathbb{G}=d_{m}$, where

$$
\begin{array}{ll}
d_{m}=d & \text { if } 1 \leq d \leq n, \\
d_{m}=d+1 & \text { if } n+1 \leq d \leq 2 n .
\end{array}
$$

Proof. It is known, and can be checked by direct computation (see e.g. [13]), that the Lebesgue measure $\mathcal{L}^{d}$ is a, left and right invariant, Haar measure on $\mathbb{G}$. Moreover, for all Borel sets $\mathcal{A} \subset \mathbb{G}$ and $r>0$,

$$
\mathcal{L}^{d}\left(\delta_{r} \mathcal{A}\right)=r^{d_{m}} \mathcal{L}^{d}(\mathcal{A})
$$

and

$$
\mathcal{L}^{d}\left\llcorner\mathbb{G}=\mathcal{H}_{\text {euc }}^{d}\llcorner\mathbb{G} .\right.
$$

Notice also that, if $\mathbb{G}$ is horizontal, then

$$
B(e, 1) \cap \mathbb{G}=B_{d_{m}, \text { euc }}
$$

and, if $\mathbb{G}$ is vertical, then

$$
B(e, 1) \cap \mathbb{G}=\left(B_{2 n, \text { euc }} \times[-1,1]\right) \cap \mathbb{G}=B_{d_{m}-2, \text { euc }} \times[-1,1] .
$$

In the preceding lines we have identified $\mathbb{G}$, respectively, with $\mathbb{R}^{d}$ or with $\mathbb{R}^{d} \times \mathbb{R}$ while $B_{m \text {,euc }}$ denotes the unit closed ball in $\mathbb{R}^{m}$. Now, let $\mathcal{A}$ be a fixed Borel set and let $p \in \mathcal{A} \cap \mathbb{G}$. Then, taking into account (2.3),

$$
\begin{aligned}
\mathcal{H}_{\text {euc }}^{d}(B(p, r) \cap \mathbb{G}) & =\mathcal{L}^{d}(B(p, r) \cap \mathbb{G})=r^{d_{m}} \mathcal{L}^{d}(B(e, 1) \cap \mathbb{G}) \\
& =r^{d_{m}} \mathcal{H}_{\text {euc }}^{d}(B(e, 1) \cap \mathbb{G})=\alpha_{d_{m}} r^{d_{m}} .
\end{aligned}
$$

Then by $[12,2.10 .17(2)$ and 2.10.19(3)]

$$
\mathcal{H}_{\mathrm{euc}}^{d}(\mathbb{G} \cap \mathcal{A})=\mathcal{S}^{d_{m}}(\mathbb{G} \cap \mathcal{A})
$$

and the thesis follows.

We say that two homogeneous subgroups are orthogonal if their subalgebras are orthogonal vector subspaces in $\mathfrak{h}$. 
Remark 2.21. If $1 \leq d \leq n$ and $\mathbb{V} \in \mathcal{G}\left(\mathbb{H}^{n}, d\right)$, there is a unique subgroup $\mathbb{V}^{\perp} \in \mathcal{G}\left(\mathbb{H}^{n}, 2 n+1-d\right)$ that is orthogonal to $\mathbb{V}$. Then $\mathbb{V}, \mathbb{V}^{\perp}$ are complementary subgroups and we define the projections

$$
P_{\mathbb{V}}: \mathbb{H}^{n} \rightarrow \mathbb{V} \text { and } P_{\mathbb{V} \perp}: \mathbb{H}^{n} \rightarrow \mathbb{V}^{\perp}
$$

where, for all $p \in \mathbb{H}^{n}, P_{\mathbb{V}}(p)$ is the $\mathbb{V}$-component of $p$ in the decomposition $\mathbb{V}, \mathbb{V}^{\perp}$ of $\mathbb{H}^{n}$ and analogously for $P_{\mathbb{V} \perp}(p)$.

As observed in Proposition 2.15, the function $P_{\mathbb{V}}$ is a homogeneous homomorphism and we can also state differently Proposition 2.15.

Proposition 2.22. Let $\mathbb{V} \in \mathcal{G}\left(\mathbb{H}^{n}, d\right)$, with $1 \leq d \leq n$. Then there is $c_{1}=c_{1}(d)>$ 0 such that (2.10) holds with $c=c_{1}$ and $p_{\mathbb{V}}=P_{\mathbb{V}}(p), p_{\mathbb{W}}=P_{\mathbb{V}^{\perp}}(p)$. Consequently, (2.11) becomes

$$
\begin{gathered}
c_{1}\left\|P_{\mathbb{V}}(p)\right\| \leq d\left(p, \mathbb{V}^{\perp}\right) \leq\left\|P_{\mathbb{V}}(p)\right\| \\
c_{1}\left\|P_{\mathbb{V}}(p)^{-1} \cdot P_{\mathbb{V} \perp}(p) \cdot P_{\mathbb{V}}(p)\right\| \leq d(p, \mathbb{V}) \leq\left\|P_{\mathbb{V}}(p)^{-1} \cdot P_{\mathbb{V} \perp}(p) \cdot P_{\mathbb{V}}(p)\right\|,
\end{gathered}
$$

for all $p \in \mathbb{H}^{n}$.

Proof. We have to check only that, if $\mathbb{W}$ is $\mathbb{V}^{\perp}$, the constant in (2.10) depends only on $d$. Indeed, by rotation, we can assume that

$$
\mathbb{V}=\left\{\left(v_{1}, \ldots, v_{d}, 0, \ldots, 0\right)\right\}
$$

hence

$$
\mathbb{W}=\mathbb{V}^{\perp}=\left\{\left(0, \ldots, 0, w_{d+1}, \ldots, w_{2 n+1}\right)\right\}
$$

and the compactness argument gives a constant depending only on $d$. Finally, (2.14) follows by the same proof giving (2.11) from (2.10).

Lemma 2.23. Let $\mathbb{V} \in \mathcal{G}\left(\mathbb{H}^{n}, d\right)$, with $1 \leq d \leq n$. Then there are $s=s(d) \in$ $(0,1)$ and $\eta=\eta(s)>0$ such that the following holds:

$$
d\left(p^{-1} \cdot q, \mathbb{V}\right)<s d(p, q) \Longrightarrow d\left(P_{\mathbb{V}}(p), P_{\mathbb{V}}(q)\right)>\eta d(p, q)
$$

whenever $p, q \in \mathbb{H}^{n}$.

Proof. Without loss of generality, we can assume $p=e$. Indeed, from (2.9),

$$
P_{\mathbb{V}}\left(p^{-1} \cdot q\right)=P_{\mathbb{V}}(p)^{-1} \cdot P_{\mathbb{V}}(q) .
$$

Hence (2.15) is equivalent to

$$
d(q, \mathbb{V})<s\|q\| \Longrightarrow\left\|P_{\mathbb{V}}(q)\right\|>\eta\|q\|,
$$

for all $q \in \mathbb{H}^{n}$. 
To prove (2.16) observe that

$$
q=P_{\mathbb{V}}(q) \cdot P_{\mathbb{V}}(q)^{-1} \cdot P_{\mathbb{V}^{\perp}}(q) \cdot P_{\mathbb{V}}(q) ;
$$

then, by triangular inequality, by the second line of (2.14) and by the assumption in (2.16), we have

$$
\begin{aligned}
\left\|P_{\mathbb{V}}(q)\right\| & \geq\|q\|-\left\|P_{\mathbb{V}}(q)^{-1} \cdot P_{\mathbb{V} \perp}(q) \cdot P_{\mathbb{V}}(q)\right\| \\
& \geq\|q\|-\frac{1}{c_{1}} d(q, \mathbb{V}) \\
& \geq\left(1-s / c_{1}\right)\|q\| .
\end{aligned}
$$

Hence, (2.15) is proved, if $s<c_{1}$, with $\eta=1-s / c_{1}$.

For $\mathcal{A} \subset \mathbb{H}^{n}$ and $r>0$, let

$$
\mathcal{A}(r)=\left\{p \in \mathbb{H}^{n}: d(p, \mathcal{A}) \leq r\right\} .
$$

Lemma 2.24. For $R>0$ and $s>0$ there is $\delta=\delta(R, s)>0$ depending only on $n, R$, such that if $\mathbb{G} \in \mathcal{G}\left(\mathbb{H}^{n}, d\right), 0<r \leq R$ and $p \in B(e, R), q_{1}, q_{2} \in B(p, r)$, with $d\left(q_{2}^{-1} \cdot q_{1}, \mathbb{G}\right) \geq s r$, then

$$
B(p, r) \cap q_{1} \cdot \mathbb{G}(\delta s r) \cap q_{2} \cdot \mathbb{G}(\delta s r)=\emptyset .
$$

Proof. Denote $B=B(e, 2 R)$. Then, by compactness,

$$
c(R, s):=\inf _{\substack{\mathbb{G} \in \mathcal{G}\left(\mathbb{H}^{n}, d\right) \\ q_{1} \in B \backslash \mathbb{G}(s R)}} d\left(B \cap q_{1} \cdot \mathbb{G}, B \cap \mathbb{G}\right)>0 .
$$

Defining $\delta=\delta(R, s)=c(R, s) /(3 s R)$, we get

$$
d\left(B \cap q_{1} \cdot \mathbb{G}, B \cap \mathbb{G}\right) \geq 3 \delta s R,
$$

this gives by triangular inequality that for $q_{1} \in B \backslash \mathbb{G}(\delta s R)$,

$$
B \cap q_{1} \cdot \mathbb{G}(\delta s R) \cap \mathbb{G}(\delta s R)=\emptyset .
$$

By translation invariance this proves the thesis in the case $r=R$ and $p=e$. The general case follows easily from this applying the translation by $p^{-1}$ and the dilation $\delta_{R / r}$.

Lemma 2.25. Let $\mathbb{V} \in \mathcal{G}\left(\mathbb{H}^{n}, d\right)$, with $1 \leq d \leq n$. Then there is $c_{2}=c_{2}(d) \geq 1$ such that, for all $p \in \mathbb{H}^{n}$,

$$
d\left(p, P_{\mathbb{V}}(p)\right) \leq c_{2} d(p, \mathbb{V}) .
$$

Moreover, for all $p \in \mathbb{H}^{n}, q \in \mathbb{V}$ and $r>0$,

$$
\operatorname{diam}\left(P_{\mathbb{V}}^{-1}(B(q, r)) \cap p \cdot \mathbb{V}(r)\right) \leq 2\left(1+c_{2}\right) r .
$$


Proof. To prove the first statement observe that, from (2.14),

$$
\begin{aligned}
d\left(p, P_{\mathbb{V}}(p)\right) & =\left\|p^{-1} \cdot P_{\mathbb{V}}(p)\right\|=\left\|P_{\mathbb{V}}(p)^{-1} \cdot P_{\mathbb{V} \perp}(p)^{-1} \cdot P_{\mathbb{V}}(p)\right\| \\
& \leq \frac{1}{c_{1}} d(p, \mathbb{V}) .
\end{aligned}
$$

Hence (2.18) follows with $c_{2}=1 / c_{1}$.

To check the second inequality, let $p_{i} \in p \cdot \mathbb{V}(r)$ with $P_{\mathbb{V}}\left(p_{i}\right) \in B(q, r)$ for $i=1,2$. Set $q_{i}=p^{-1} \cdot p_{i}$. Then $d\left(q_{i}, \mathbb{V}\right) \leq r$ and, as $P_{\mathbb{V}}$ is a homomorphism, $d\left(P_{\mathbb{V}}\left(q_{1}\right), P_{\mathbb{V}}\left(q_{2}\right)\right)=d\left(P_{\mathbb{V}}\left(p_{1}\right), P_{\mathbb{V}}\left(p_{2}\right)\right) \leq 2 r$. So, by $(2.18)$,

$$
\begin{aligned}
d\left(p_{1}, p_{2}\right) & =d\left(q_{1}, q_{2}\right) \\
& \leq d\left(q_{1}, P_{\mathbb{V}}\left(q_{1}\right)\right)+d\left(P_{\mathbb{V}}\left(q_{1}\right), P_{\mathbb{V}}\left(q_{2}\right)\right)+d\left(q_{2}, P_{\mathbb{V}}\left(q_{2}\right)\right) \\
& \leq 2\left(1+c_{2}\right) r .
\end{aligned}
$$

This proves the lemma.

The last lemma is false for $n<d \leq 2 n$; the second inequality only holds with $r$ replaced by $C \sqrt{r}$. This is the main obstacle for carrying out the proof of the main theorem without the positive lower density assumption in the case $n<d \leq 2 n$.

\subsection{Intrinsic cones}

We begin with the definition of intrinsic cone in $\mathbb{H}^{n}$.

Definition 2.26. Let $\mathbb{G}$ be a d-subgroup of $\mathbb{H}^{n}$. The intrinsic cone $X\left(p_{0}, \mathbb{G}, s\right)$, with vertex $p_{0} \in \mathbb{H}^{n}$, axis $\mathbb{G}$ and opening $s \in(0,1)$, is

$$
X\left(p_{0}, \mathbb{G}, s\right):=\left\{p \in \mathbb{H}^{n}: d\left(p_{0}^{-1} \cdot p, \mathbb{G}\right)<s d\left(p, p_{0}\right)\right\} .
$$

This definition is invariant by group translations, that is,

$$
\begin{aligned}
X\left(p_{0}, \mathbb{G}, s\right) & =p_{0} \cdot X(e, \mathbb{G}, s) \\
& =p_{0} \cdot\left\{p \in \mathbb{H}^{n}: d(p, \mathbb{G})<s d(p, e)\right\} .
\end{aligned}
$$

If $v \in \mathfrak{h}_{1}$ we denote by $\mathbb{N}(v) \in \mathcal{G}\left(\mathbb{H}^{n}, 2 n\right)$ the 1-codimensional normal subgroup orthogonal to $v$, that is,

$$
\mathbb{N}(v):=\left\{p \in \mathbb{H}^{n}:\langle\nu, \pi(p)\rangle=0\right\} .
$$

Proposition 2.27. If $v \in \mathfrak{h}_{1}$ and $\|v\|=1$, then, for all $p_{0} \in \mathbb{H}^{n}$ and for all $s \in(0,1)$,

$$
X\left(p_{0}, \mathbb{N}(v), s\right)=\left\{p \in \mathbb{H}^{n}:\left|\left\langle\nu, \pi\left(p_{0}^{-1} \cdot p\right)\right\rangle\right|<s d\left(p, p_{0}\right)\right\} .
$$


Proof. Given (2.19), we assume $p_{0}=e$. Then, because

$$
\begin{aligned}
d(p, \mathbb{N}(v)) & =\inf \{d(p, q): q \in \mathbb{N}(v)\}=\inf \left\{\left|p^{\prime}-q^{\prime}\right|_{\mathbb{R}^{2 n}}: q \in \mathbb{N}(v)\right\} \\
& =\left|\sum_{i=1}^{2 n} p_{i} v_{i}\right| /\|v\|=\left|\sum_{i=1}^{2 n} p_{i} v_{i}\right| ;
\end{aligned}
$$

it follows that $d(p, \mathbb{N}(v))=|\langle v, \pi(p)\rangle|$.

Notice that an $\mathbb{H}$-regular submanifold $S$ is locally contained, at any point $p \in S$, in a cone with axis the tangent group $T_{\mathbb{H}} S(p)$.

Lemma 2.28. Let $S \subset \mathbb{H}^{n}$ be a d-dimensional $\mathbb{H}$-regular surface, let $p_{0} \in S$. Then, for all $\alpha>0$, there exists $\bar{r}=\bar{r}\left(S, p_{0}, \alpha\right)>0$ such that

$$
U\left(p_{0}, \bar{r}\right) \cap S \subset X\left(p_{0}, T_{\mathbb{H}} S\left(p_{0}\right), \alpha\right) .
$$

Proof. We divide the proof in two cases.

Assume $1 \leq d \leq n$. By Definitions 2.10 and 2.11, there are $r>0$ and an injective continuously Pansu differentiable function $\varphi: \mathcal{U} \subset \mathbb{R}^{d} \rightarrow \mathbb{H}^{n}$ such that

$$
S \cap U\left(p_{0}, r\right)=\{\varphi(x): x \in \mathcal{U}\}, \quad T_{\mathbb{H}} S\left(p_{0}\right)=\left\{d_{H} \varphi_{x_{0}}(x): x \in \mathbb{R}^{d}\right\},
$$

where $p_{0}=\varphi\left(x_{0}\right)$. Let $p=\varphi(x)$, then

$$
\begin{aligned}
& d\left(p_{0}^{-1} \cdot p, T_{\mathbb{H}} S\left(p_{0}\right)\right) \\
& \quad \leq\left\|d_{H} \varphi_{x_{0}}\left(x_{0}-x\right) \cdot \varphi\left(x_{0}\right)^{-1} \cdot \varphi(x)\right\|=o\left(\left|x_{0}-x\right|_{\mathbb{R}^{d}}\right) .
\end{aligned}
$$

Since $d_{H} \varphi_{x_{0}}: \mathbb{R}^{d} \rightarrow \mathbb{H}^{n}$ is H-linear and injective, there is $c=c\left(p_{0}, \varphi\right)>0$ such that

$$
\left\|d_{H} \varphi_{x_{0}}\left(x_{0}-x\right)\right\| \geq c\left|x_{0}-x\right|_{\mathbb{R}^{d}} .
$$

Hence, for $\left|x_{0}-x\right|_{\mathbb{R}^{d}}$ small,

$$
\begin{aligned}
\left\|p_{0}^{-1} \cdot p\right\| & \geq\left\|d_{H} \varphi_{x_{0}}\left(x-x_{0}\right)\right\|-d\left(d_{H} \varphi_{x_{0}}\left(x-x_{0}\right), p_{0}^{-1} \cdot p\right) \\
& \geq(c / 2)\left|x_{0}-x\right|_{\mathbb{R}^{d}} .
\end{aligned}
$$

From (2.22) and (2.23) we get (2.21).

Assume $n+1 \leq d \leq 2 n$. Once more by Definitions 2.10 and 2.11, there are $r>0$ and $f \in\left[\mathbf{C}_{\mathbb{H}}^{1}\left(\bar{U}\left(p_{0}, r\right)\right)\right]^{2 n+1-d}$ such that $d_{H} f_{p_{0}}: \mathbb{H}^{n} \rightarrow \mathbb{R}^{2 n+1-d}$ is surjective and

$$
\begin{aligned}
S \cap U\left(p_{0}, r\right) & =\{p: f(p)=0\} \\
T_{\mathbb{H}} S\left(p_{0}\right) & =\operatorname{ker}\left(d_{H} f_{p_{0}}\right) .
\end{aligned}
$$


If $p \in S \cap U\left(p_{0}, r\right)$ from (2.7) we have that

$$
\left|d_{H} f_{p_{0}}\left(p_{0}^{-1} \cdot p\right)\right|_{\mathbb{R}^{2 n+1-d}}=o\left(d\left(p_{0}, p\right)\right) .
$$

On the other hand, since $d_{H} f_{p_{0}}: \mathbb{H}^{n} \rightarrow \mathbb{R}^{2 n+1-d}$ is H-linear, there is $c=$ $c\left(p_{0}, f\right)>0$ such that

$$
\left|d_{H} f_{p_{0}}\left(p_{0}^{-1} \cdot p\right)\right|_{\mathbb{R}^{2 n+1-d}} \geq c d\left(p_{0}^{-1} \cdot p, \operatorname{ker}\left(d_{H} f_{p_{0}}\right)\right) .
$$

Indeed, if $L: \mathbb{H}^{n} \rightarrow \mathbb{R}^{2 n+1-d}, n+1 \leq d \leq 2 n$, is H-linear then $\operatorname{ker}(L)$ is a vertical subgroup of $\mathbb{H}^{n}$ and (by Lemma 3.26 of [17]) there is a horizontal $(2 n+1-d)$ subgroup $\mathbb{V}$ such that $\mathbb{H}^{n}=\operatorname{ker}(L) \cdot \mathbb{V}$. Then $L: \mathbb{V} \rightarrow \mathbb{R}^{2 n+1-d}$ is injective and there exists $c>0$ such that

$$
|L(v)|_{\mathbb{R}^{2 n+1-d}} \geq c\|v\|
$$

for all $v \in \mathbb{V}$.

From (2.24) and (2.25) we get (2.21).

Now we collect a few technical Lemmas that will be used in the proofs of the main results.

Lemma 2.29. Let $\mathbb{G}$ be a homogeneous subgroup of $\mathbb{H}^{n}$ and $s \in(0,1]$. Then, for $p \in \mathbb{H}^{n}$ and $q \in \mathbb{H}^{n} \backslash X(p, \mathbb{G}, s)$,

$$
B\left(q, \frac{s}{2} d(p, q)\right) \subset \mathbb{H}^{n} \backslash X(p, \mathbb{G}, s / 3) .
$$

Proof. Let $r=d(p, q)>0$ and $z \in B(q, s r / 2)$. Then $d(z, p) \leq s r / 2+r \leq 3 r / 2$. If $g \in \mathbb{G}$, then

$$
\begin{aligned}
d\left(p^{-1} \cdot z, g\right) & \geq d\left(p^{-1} \cdot q, g\right)-d\left(p^{-1} z, p^{-1} \cdot q\right) \\
& \geq s d(p, q)-d(z, q) \\
& \geq s r-s r / 2=s r / 2 \geq s d(z, p) / 3
\end{aligned}
$$

whence $d\left(p^{-1} z, \mathbb{G}\right) \geq s d(z, p) / 3$ and $z \notin X(p, \mathbb{G}, s / 3)$, which proves the lemma.

Lemma 2.30. If $1 \leq d \leq 2 n$ and $0<s \leq 1$, there are $k=k(s)$ homogeneous subgroups of $\mathbb{H}^{n}, \mathbb{G}_{1}, \ldots, \mathbb{G}_{k}$, with the same dimension $d$ and metric dimension $d_{m}$ such that for all $p \in \mathbb{H}^{n}$ and for all homogeneous subgroups $\mathbb{G}$ with dimension $d$ and metric dimension $d_{m}$

$$
X(p, \mathbb{G}, s) \subset X\left(p, \mathbb{G}_{i}, 3 s\right), \text { for some } i=1, \ldots, k .
$$

Proof. By a simple compactness argument, recalling Remark 2.18 , we find $\mathbb{G}_{1}, \ldots, \mathbb{G}_{k}$ such that for all $\mathbb{G}, \rho\left(\mathbb{G}, \mathbb{G}_{i}\right)<s$ for some $i=1, \ldots, k$. The inclusion follows easily from this, by definition of $X(p, \mathbb{G}, s)$. 
Lemma 2.31. Let $\mathbb{G}_{1}, \mathbb{G}_{2}$ be homogeneous subgroups of $\mathbb{H}^{n}$, with dimension $d$ and metric dimension $d_{m}$. Assume $\mathbb{G}_{1} \neq \mathbb{G}_{2}$ and let $\bar{d}=\operatorname{dim}\left(\mathbb{G}_{1} \cap \mathbb{G}_{2}\right)$ and $\bar{d}_{m}$ be the metric dimension of $\mathbb{G}_{1} \cap \mathbb{G}_{2}$. Then $0 \leq \bar{d} \leq d-1$ and, for $0<s<1$, there is $\delta(s)>0$ such that $\lim _{s \rightarrow 0} \delta(s)=0$ and the following holds:

for all $p \in \mathbb{H}^{n}, 0<r<\infty$ and $0<s<1$,

$$
B(p, r) \cap X\left(p, \mathbb{G}_{1}, s\right) \cap X\left(p, \mathbb{G}_{2}, s\right)
$$

can be covered with $k$ balls of radius $\delta(s) r$, where $k \leq C \delta(s)^{-\bar{d}_{m}}$ and $C=C(n, \bar{d})$.

Proof. We can assume that $p=e$ and $r=1$. Since $\mathbb{G}_{1} \neq \mathbb{G}_{2}$, then $0 \leq \bar{d}=$ $\operatorname{dim}\left(\mathbb{G}_{1} \cap \mathbb{G}_{2}\right) \leq d-1$. It follows that, for any $0<t \leq 1, B(e, 1) \cap \mathbb{G}_{1} \cap \overline{\mathbb{G}}_{2}$ can be covered with $C_{1} t^{-\bar{d}_{m}}$ balls of radius $t$ where $C_{1}$ depends only on $n$ and $\bar{d}_{m}$. Next we observe that there is $\delta(s)>0$ with $\lim _{s \rightarrow 0} \delta(s)=0$ such that

$$
B(e, 1) \cap X\left(e, \mathbb{G}_{1}, s\right) \cap X\left(e, \mathbb{G}_{2}, s\right) \subset\left(\mathbb{G}_{1} \cap \mathbb{G}_{2}\right)(\delta(s) / 2),
$$

where $A(r)$ is defined in 2.17. If this were not true, we could find $\eta>0, q_{j} \in$ $B(e, 1), j=1,2 \ldots$, such that $d\left(q_{j}, \mathbb{G}_{i}\right) \leq d\left(q_{j}, e\right) / j \leq 1 / j, i=1,2$, and $d\left(q_{j}, \mathbb{G}_{1} \cap \mathbb{G}_{2}\right) \geq \eta$. By going to a suitable subsequence, we could also assume that $q_{j} \rightarrow q$. Then $q \in \mathbb{G}_{1} \cap \mathbb{G}_{2}$ and $d\left(q, \mathbb{G}_{1} \cap \mathbb{G}_{2}\right) \geq \eta$, which is impossible. Combining the above two facts with $t=\delta(s) / 2$ we see that the lemma holds with $C=C_{1} 2^{\bar{d}_{m}} \leq C_{1} 2^{d}$.

Lemma 2.32. Let $\mathbb{W} \in \mathcal{G}\left(\mathbb{H}^{n}, d\right)$ with $n<d \leq 2 n$. Then there are unit vectors $v_{1}, \cdots, v_{2 n+1-d} \in \mathfrak{h}_{1}$, such that

$$
\mathbb{V}:=\exp \left(\operatorname{span}\left\{v_{1}, \cdots, v_{2 n+1-d}\right\}\right) \in \mathcal{G}\left(\mathbb{H}^{n}, 2 n+1-d\right),
$$

is (a horizontal subgroup) complementary to $\mathbb{W}$. The vectors $v_{j}$ can be chosen continuously dependent on $\mathbb{W}$ in sufficiently small neighborhoods in $\mathcal{G}\left(\mathbb{H}^{n}, d\right)$. Moreover, $\mathbb{W}=\bigcap_{j=1}^{2 n+1-d} \mathbb{N}\left(v_{j}\right)$, and, for all $p \in \mathbb{H}^{n}$,

$$
X(p, \mathbb{W}, s) \subset \bigcap_{i=1}^{2 n+1-d} X\left(p, \mathbb{N}\left(v_{i}\right), s\right)
$$

Proof. The existence of a horizontal subgroup $\mathbb{V}$, complementary to $\mathbb{W}$, is the content of [17, Lemma 3.26]. Moreover, from the proof of that lemma, it follows that $\mathbb{V}$ depends continuously on $\mathbb{W}$. Hence, we can choose $v_{1}, \cdots, v_{2 n+1-d}$ as an orthonormal basis of $\mathbb{V}$, continuously dependent on $\mathbb{W}$.

Let $\mathfrak{w} \subset \mathfrak{h}$ be the Lie algebra of $\mathbb{W}$ and let $\mathfrak{w}_{i}$ be the subalgebra generated by $\mathfrak{w}, v_{1}, \ldots, v_{i}, v_{i+1}, \ldots, v_{2 n+1-d}$, for $i=1, \ldots, 2 n+1-d$. Each $\mathfrak{w}_{i}$ is a subalgebra of $\mathfrak{h}$, hence $\mathbb{N}\left(v_{i}\right)=\exp \left(\mathfrak{w}_{i}\right) \in \mathcal{G}\left(\mathbb{H}^{n}, 2 n\right)$ and $\mathbb{W}=\bigcap_{i} \mathbb{N}\left(v_{i}\right)$. Finally, because $d\left(p, \mathbb{N}\left(v_{i}\right)\right) \leq d(p, \mathbb{W})$, also $X(p, \mathbb{W}, s) \subset X\left(p, \mathbb{N}\left(v_{i}\right), s\right)$ and (2.26) follows. 


\section{Rectifiable sets and measures in $\mathbb{H}^{n}$}

\subsection{Measures, densities and tangent measures}

Let $\mu$ be an outer measure in $\mathbb{H}^{n}$. The image $f_{\#} \mu$ under a map $f: \mathbb{H}^{n} \rightarrow \mathbb{H}^{n}$ is the measure on $\mathbb{H}^{n}$ defined by

$$
f_{\#} \mu(\mathcal{A})=\mu\left(f^{-1}(\mathcal{A})\right), \quad \text { for all } \mathcal{A} \subset \mathbb{H}^{n} .
$$

For $a \in \mathbb{H}^{n}$ and $r>0, T_{a, r}: \mathbb{H}^{n} \rightarrow \mathbb{H}^{n}$ is defined, for all $p \in \mathbb{H}^{n}$, as

$$
T_{a, r}(p):=\delta_{1 / r}\left(a^{-1} \cdot p\right) .
$$

Definition 3.1. Let $\mu$ be a Radon measure on $\mathbb{H}^{n}$. We say that $v$ is a tangent measure of $\mu$ at $a \in \mathbb{H}^{n}$ if $v$ is a Radon measure on $\mathbb{H}^{n}$ with $v\left(\mathbb{H}^{n}\right)>0$ and there are positive numbers $c_{i}$ and $r_{i}, i=1,2, \ldots$, such that $r_{i} \rightarrow 0$ and

$$
c_{i} T_{a, r_{i} \#} \mu \rightarrow v, \quad \text { weakly as } i \rightarrow \infty .
$$

We denote by $\operatorname{Tan}(\mu, a)$ the set of all tangent measures of $\mu$ at a.

The numbers $c_{i}$ are normalization constants which are needed to keep $v$ nontrivial and locally finite. Often, as below, one can use $c_{i}=\mu\left(B\left(a, r_{i}\right)\right)^{-1}$.

Definition 3.2. Let $\mu$ be a Radon measure on $\mathbb{H}^{n}$. We say that $\mu$ has a unique tangent measure $v$ at $a$ if $v$ is a Radon measure on $\mathbb{H}^{n}$ such that

$$
\operatorname{Tan}(\mu, a)=\{c v: 0<c<\infty\} .
$$

Of course, such a $v$ is unique only up to multiplication by positive constants.

The following theorem was recently proved in [24] in a much more general setting, i.e., inside locally compact metric groups with dilations.

Theorem 3.3. Let $\mu$ be a Radon measure on $\mathbb{H}^{n}$. Then the following two conditions are equivalent:

(1) $\mu$ has a unique tangent measure $v_{a}$ at $\mu$-a.e. $a \in \mathbb{H}^{n}$.

(2) For $\mu$-a.e. a $\in \mathbb{H}^{n}$ there exists a closed homogeneous subgroup $\mathbb{G}_{a}$ of $\mathbb{H}^{n}$ for which

$$
\operatorname{Tan}(\mu, a)=\left\{c \lambda_{a}: 0<c<\infty\right\}
$$

where $\lambda_{a}$ is a left Haar measure of $\mathbb{G}_{a}$.

Moreover, if these conditions hold, it is possible to choose $\lambda_{a}$ such that

$$
\frac{1}{\mu(B(a, r))} T_{a, r \# \mu} \rightarrow \lambda_{a}, \quad \text { weakly as } r \rightarrow 0 .
$$


Notice that the subgroups $\mathbb{G}_{a}$ of Theorem 3.3 are not necessarily in $\mathcal{G}\left(\mathbb{H}^{n}\right)$. The easiest example being $\mu:=\mathcal{S}^{2}\llcorner\mathbb{T}$ : in this case, $\mu$ is invariant by dilations and $\mu$ is its own tangent measure at any point $a \in \mathbb{T}$. More generally, if $\gamma \subset \mathbb{H}^{1}$ is any non horizontal $C^{1}$ curve, and $\mu:=\mathcal{S}^{2}\llcorner\gamma$, then once more the unique tangent measure to $\mu$ in any point $a \in \gamma$ is the measure $v:=\mathcal{S}^{2}\llcorner\mathbb{T}$.

Any measure $\mathcal{S}^{d_{m}}\llcorner S$, with $S \mathbb{H}$-regular surface, has, at each point $p \in S$, a unique tangent measure in the sense of Definition 3.2; this tangent measure is always supported on $T_{\mathbb{H}} S(p)$, hence it is supported on a subgroup in $\mathcal{G}\left(\mathbb{H}^{n}\right)$ (see $[14,16,26])$. Indeed we have

Theorem 3.4. Let $S \subset \mathbb{H}^{n}$ be a d-dimensional $\mathbb{H}$-regular surface. Then $S$ has Hausdorff dimension $d_{m}$ (remember (2.13)) and, denoting

$$
S_{p_{0}, r}:=\left\{p \in \mathbb{H}^{n}: p_{0} \cdot \delta_{r}\left(p_{0}^{-1} \cdot p\right) \in S\right\},
$$

for $p_{0} \in \mathbb{H}^{n}$ and $r>0$, we have

$$
\begin{aligned}
\lim _{r \rightarrow 0} \frac{\mathcal{S}^{d_{m}}\left(S_{p_{0}, r} \cap U(0,1)\right)}{\alpha_{d_{m}} r^{d_{m}}} & =\frac{\mathcal{S}^{d_{m}}\left(T_{\mathbb{H}} S\left(p_{0}\right) \cap U(0,1)\right)}{\alpha_{d_{m}}} \\
& =\frac{\mathcal{H}_{\mathrm{euc}}^{d}\left(T_{\mathbb{H}} S\left(p_{0}\right) \cap U(0,1)\right)}{\alpha_{d_{m}}}=1 .
\end{aligned}
$$

Moreover,

$$
\frac{1}{r^{d_{m}}} T_{p_{0}, r \#}\left(\mathcal { S } ^ { d _ { m } } \llcorner S ) \rightarrow \mathcal { S } ^ { d _ { m } } \left\llcornerT_{\mathbb{H}} S\left(p_{0}\right),\right.\right.
$$

as $r \rightarrow 0$, weakly in the sense of measures, whence

$$
\operatorname{Tan}\left(\mathcal{S}^{d_{m}}\left\llcorner S, p_{0}\right)=\left\{c \mathcal{S}^{d_{m}}\left\llcorner T_{\mathbb{H}} S\left(p_{0}\right): 0<c<\infty\right\} .\right.\right.
$$

Following [23] we define

Definition 3.5. Let $\mu$ be a Radon measure in $\mathbb{H}^{n}$ and $m>0$. We define the upper and lower $m$-densities of $\mu$ at $p \in \mathbb{H}^{n}$ as

$$
\Theta^{* m}(\mu, p):=\limsup _{r \rightarrow 0} \frac{\mu(B(p, r))}{\alpha_{m} r^{m}}
$$

and

$$
\Theta_{*}^{m}(\mu, p):=\liminf _{r \rightarrow 0} \frac{\mu(B(p, r))}{\alpha_{m} r^{m}},
$$

where $\alpha_{m}$ is defined in (2.3). In particular, if $E \subset \mathbb{H}^{n}$ is $\mathcal{S}^{m}$ measurable with $\mathcal{S}^{m}\left\llcorner E\right.$ locally finite, we define the upper and lower $m$-densities of $E$ at $p \in \mathbb{H}^{n}$ as

$$
\Theta^{* m}(E, p):=\limsup _{r \rightarrow 0} \frac{\mathcal{S}^{m}(E \cap B(p, r))}{\alpha_{m} r^{m}}
$$


and

$$
\Theta_{*}^{m}(E, p):=\liminf _{r \rightarrow 0} \frac{\mathcal{S}^{m}(E \cap B(p, r))}{\alpha_{m} r^{m}} .
$$

If they agree their common value

$$
\Theta^{m}(E, p)=\Theta^{* m}(E, p)=\Theta_{*}^{m}(E, p)
$$

is called the $m$-density of $E$ at $p$.

Lemma 3.6. Let $E \subset \mathbb{H}^{n}$ be $\mathcal{S}^{m}$ measurable with $\mathcal{S}^{m}(E)<\infty$. Then

(i) $2^{-m} \leq \Theta^{* m}(E, p) \leq 1$, for $\mathcal{S}^{m}$-a.e. $p \in E$;

(ii) $\Theta^{* m}(E, p)=0$, for $\mathcal{S}^{m}$-a.e. $p \in \mathbb{H}^{n} \backslash E$;

Proof. All the statements follow from general results on densities of spherical Hausdorff measures in metric spaces (see [23, Theorem 6.2] and [12, 2.10.19]).

Following [23] we define the approximate tangent group $\operatorname{apTan}_{\mathbb{H}}^{d}(E, p)$ of $E$ at $p \in \mathbb{H}^{n}$, as follows

Definition 3.7. Let $E \subset \mathbb{H}^{n}$ be $\mathcal{S}^{d_{m}}$ measurable. We say that the homogeneous subgroup $\mathbb{T}_{p}$, of dimension $d$ and metric dimension $d_{m}$, is a $(d, \mathbb{H})$-approximate tangent group to $E$ at $p$ if $\Theta^{* d_{m}}(E, p)>0$ and

$$
\lim _{r \rightarrow 0} \frac{\mathcal{S}^{d_{m}}\left(E \cap B(p, r) \backslash X\left(p, \mathbb{T}_{p}, s\right)\right)}{r^{d_{m}}}=0,
$$

for all $0<s<1$. We write $\operatorname{apTan}_{\mathbb{H}}^{d}(E, p)$ for the set of all $(d, \mathbb{H})$-approximate tangent groups to $E$ at $p$. If there is only one subgroup $\mathbb{T}_{p} \in \operatorname{apTan}_{\mathbb{H}}^{d}(E, p)$ we write $\mathbb{T}_{p}=\operatorname{apTan}_{\mathbb{H}}^{d}(E, p)$.

Remark 3.8. Let $1 \leq d \leq n, B$ be a Borel subset of $\mathbb{R}^{d}$ and $f: B \rightarrow \mathbb{H}^{n}$ be a Lipschitz function. If $E=f(B)$ and if $f$ is Pansu differentiable at $x_{0} \in B$ with injective Pansu differential $d_{H} f_{x_{0}}$ then, at $p_{0}=f\left(x_{0}\right)$,

$$
\operatorname{apTan}_{\mathbb{H}}^{d}\left(E, p_{0}\right)=d_{H} f_{x_{0}}\left(\mathbb{R}^{d}\right)=\operatorname{Tan}^{d}\left(E, p_{0}\right),
$$

where $\operatorname{Tan}^{d}\left(E, p_{0}\right)$ is the approximate tangent space introduced in [2, Definition 5.5], and, see Proposition 2.8,

$$
f\left(x_{0}\right) \cdot \operatorname{apTan}_{\mathbb{H}}^{d}\left(E, p_{0}\right)=f\left(x_{0}\right)+\operatorname{Tan}^{d}\left(E, p_{0}\right) .
$$

The proof of the first equality is given in the first part of the proof of Lemma 2.28. The second equality follows from the following considerations. 
If $f: B \subset \mathbb{R}^{d} \rightarrow \mathbb{H}^{n}$ is Lipschitz and if $B$ is bounded then $f$ is also Euclidean Lipschitz from $B$ to $\mathbb{R}^{2 n+1}$. For a Euclidean Lipschitz function $f$, in a differentiability point $x_{0}$ of $f$, we have

$$
\operatorname{Tan}^{d}\left(f(B), f\left(x_{0}\right)\right)=d f_{x_{0}}\left(\mathbb{R}^{d}\right) .
$$

where $\operatorname{Tan}^{d}\left(f(B), f\left(x_{0}\right)\right)$ is the approximate tangent space of [2] and $d f_{x_{0}}$ is the usual differential of $f$. Then the second equality follows from Proposition 2.8.

Finally notice that, for $1 \leq d \leq n$, also the tangent group $T_{\mathbb{H}} S(p)$ to an $\mathbb{H}-$ regular surface $S$ coincides with the approximate tangent space $\operatorname{Tan}^{d}(S, p)$.

Approximate tangent groups are unique almost everywhere. Indeed we have:

Proposition 3.9. Let $E \subset \mathbb{H}^{n}$ be $\mathcal{S}^{d_{m}}$ measurable, with $\mathcal{S}^{d_{m}}(E)<\infty$, and let $A$ be the set of those $p \in E$ for which $E$ has some $(d, \mathbb{H})$-approximate tangent subgroup at $p$ of metric dimension $d_{m}$. Then

(i) $A$ is $\mathcal{S}^{d_{m}}$ measurable,

(ii) E has a unique $(d, \mathbb{H})$-approximate tangent subgroup $\mathbb{G}_{p}$ at $\mathcal{S}^{d_{m}}$-a.e. $p \in A$,

(iii) the mapping $p \mapsto \mathbb{G}_{p}$ is $\mathcal{S}^{d_{m}}$ measurable.

Proof. By Borel regularity we may assume that $E$ is a Borel set. We shall show that then $A$ is a Suslin set (see [12, Section 2.2] for the required facts on Suslin sets). Define for $r>0, i=1,2 \ldots, p \in E$ and $\mathbb{G} \in \mathcal{H}\left(\mathbb{H}^{n}, d, d_{m}\right)$,

$$
\begin{gathered}
f_{i, r}(p, \mathbb{G})=r^{-d_{m}} \mathcal{S}^{d_{m}}(E \cap B(p, r) \backslash X(p, \mathbb{G}, 1 / i)), \\
f_{i}(p, \mathbb{G})=\limsup _{r \rightarrow 0} f_{i, r}(p, \mathbb{G}) .
\end{gathered}
$$

One checks easily that $f_{i, r}$ is upper semicontinuous and the upper limit can be taken through rational values of $r$, whence $f_{i}$ is a Borel function. Let $B$ be the set of those $(p, \mathbb{G}) \in E \times \mathcal{H}\left(\mathbb{H}^{n}, d, d_{m}\right)$ such that $\mathbb{G}$ is a $(d, \mathbb{H})$-approximate tangent subgroup of $E$ at $p$. Then

$$
B=\bigcap_{i=1}^{\infty}\left\{(p, \mathbb{G}): f_{i}(p, \mathbb{G})=0\right\},
$$

and so $B$ is a Borel set. Clearly $A$ is the projection on $E$ of $B$, consequently $A$ is a Suslin set. This proves (i).

For Borel sets $F \subset E$ we have, for $\mathcal{S}^{d_{m}}$-a.e. $p \in F$, that $\mathbb{G}$ is a $(d, \mathbb{H})$ approximate tangent subgroup of $F$ at $p$ if and only is $\mathbb{G}$ is a $(d, \mathbb{H})$-approximate tangent subgroup of $E$ at $p$. This follows from the fact that $\Theta^{* d_{m}}(E \backslash F, p)=0$ for $\mathcal{S}^{d_{m}}$-a.e. $p \in F$, recall Lemma 3.6. Since $\Theta^{* d_{m}}(E, p) \leq 1$ for $\mathcal{S}^{d_{m}}$-a.e. $p \in F$, we may assume that

$$
\mathcal{S}^{d_{m}}(E \cap B(p, r)) \leq 2 \alpha_{d_{m}} r^{d_{m}} \text { for all } p \in E, r>0,
$$


whence

$$
\mathcal{S}^{d_{m}}(E \cap B(p, r)) \leq 2^{1+d_{m}} \alpha_{d_{m}} r^{d_{m}} \text { for all } p \in \mathbb{H}^{n}, r>0,
$$

by decomposing $E$ as a countable union of sets of this type and a set of measure 0 .

By Lemma 2.31 for $\mathbb{G}_{1}, \mathbb{G}_{2} \in \mathcal{H}\left(\mathbb{H}^{n}, d, d_{m}\right)$ with $\mathbb{G}_{1} \neq \mathbb{G}_{2}$ and for $0<s<$ 1 , there is $\delta(s)>0$ such that $\lim _{s \rightarrow 0} \delta(s)=0$ and the following holds: for all $p \in \mathbb{H}^{n}, 0<r<\infty$ and $0<s<1, B(p, r) \cap X\left(p, \mathbb{G}_{1}, s\right) \cap X\left(p, \mathbb{G}_{2}, s\right)$ can be covered with $k$ balls of radius $\delta(s) r$ where $k \leq C \delta(s)^{1-d_{m}}$. Then by (3.3)

$$
\mathcal{S}^{d_{m}}\left(E \cap B(p, r) \cap X\left(p, \mathbb{G}_{1}, s\right) \cap X\left(p, \mathbb{G}_{2}, s\right)\right) \leq 2^{1+d_{m}} C \delta(s) \alpha_{d_{m}} r^{d_{m}} .
$$

If $\mathbb{G}_{1}$ and $\mathbb{G}_{2}$ are $(d, \mathbb{H})$-approximate tangent subgroups of $E$ at $p$, then

$$
\lim _{r \rightarrow 0} r^{-d_{m}} \mathcal{S}^{d_{m}}\left(E \cap B(p, r) \backslash\left(X\left(p, \mathbb{G}_{1}, s\right) \cap X\left(p, \mathbb{G}_{2}, s\right)\right)\right)=0,
$$

so $\Theta^{* d_{m}}(E, p) \leq 2^{1+d_{m}} C \delta(s)$. This concludes the proof of (ii), since by Lemma 3.6, $\Theta^{* d_{m}}(E, p) \geq 2^{-d_{m}}$ for $\mathcal{S}^{d_{m}}$-a.e. $p \in E$.

By (i) and (ii) the set $C$ of those $p \in A$ for which $E$ has a unique $(d, \mathbb{H})$ approximate tangent subgroup $\mathbb{G}_{p}$ at $a$ is $\mathcal{S}^{d_{m}}$ measurable. So to prove (iii), it is enough to show that for every Borel set $D \subset C$ the map $p \mapsto \mathbb{G}_{p}, p \in D$, is Borel measurable. Let $F=B \cap\left(D \times \mathcal{H}\left(\mathbb{H}^{n}, d, d_{m}\right)\right)$ where $B$ is the Borel set of the proof of (i). Then $F$ is a Borel set and the projection $\Pi: F \rightarrow D$ is 1-1. Thus $\Pi(H)$ is a Borel set for every Borel set $H \subset F$, (see [12], p. 67). Let $f=\Pi^{-1}: D \rightarrow F$, that is $f(p)=\left(p, \mathbb{G}_{p}\right)$. Then for every Borel set $H \subset F, f^{-1}(H)=\Pi(H)$ is a Borel set and so $f$ is Borel measurable. Thus also $p \mapsto \mathbb{G}_{p}, p \in D$, is Borel measurable.

We shall now give a general result in the spirit that approximate tangent group properties imply positive lower density. This will allow us to prove the main result without positive lower density assumption in low dimensions.

Theorem 3.10. Let $1 \leq d \leq n$. There exists $\eta, 0<\eta<1$, depending only on $n$ and $d$ with the following property. Let $E \subset \mathbb{H}^{n}$ be $\mathcal{S}^{d}$ measurable with $\mathcal{S}^{d}(E)<\infty$ such that for $\mathcal{S}^{d}$-a.e. $p \in E$ there exists a horizontal subgroup $\mathbb{V}_{p} \in \mathcal{G}\left(\mathbb{H}^{n}, d\right)$ for which

$$
\lim _{r \rightarrow 0} r^{-d} \mathcal{S}^{d}\left(E \cap B(p, r) \backslash X\left(p, \mathbb{V}_{p}, \eta\right)\right)=0
$$

Then

$$
\theta_{*}^{d}(E, p)>0
$$

for $\mathcal{S}^{d}$-a.e. $p \in E$.

Proof. Denote $\mu=\mathcal{S}^{d}\left\llcorner E\right.$. Then, by Lemma 3.6, for $\mathcal{S}^{d}$-a.e. $p \in E$,

$$
\theta^{* d}(\mu, p) \geq 2^{-d} .
$$


Let $A$ be a Borel subset of $E$ such that for $p \in A$ (3.6) and (3.4) hold and

$$
\theta_{*}^{d}(\mu, p)=0 .
$$

We need to show that $\mu(A)=0$. By decomposing $\mu$ almost all of $A$ into countable unions we shall make various reductions of the problem. First, by (3.6), we may assume that

$$
\theta^{* d}(\mu, p)>2^{-d-1} \text { for } p \in A .
$$

Let $c_{2}$ be as in Lemma 2.25 and let $C=2\left(1+c_{2}\right) d, c=\left(2^{6} \cdot C\right)^{-d} \alpha_{d}, s=$ $(2 C)^{-1}, \delta=\delta(1 / 2, s)>0$ as in Lemma 2.24 and $\eta=\delta s / 6$. Let $0<\epsilon<c$. We can assume that $A \subset B(e, 1 / 2)$. By Lemma 2.30 we may assume that there is $\mathbb{V} \in \mathcal{G}\left(\mathbb{H}^{n}, d\right)$ such that for $p \in A$,

$$
X\left(p, \mathbb{V}_{p}, \delta s / 6\right) \subset X(p, \mathbb{V}, \delta s / 2),
$$

whence for $r>0$,

$$
B(p, r) \cap X\left(p, \mathbb{V}_{p}, \eta\right) \subset\left\{q \in \mathbb{H}^{n}: d(q, p \cdot \mathbb{V})<\delta s r / 2\right\}=p \cdot \mathbb{V}(\delta s r / 2),
$$

and so by (3.4)

$$
\lim _{r \rightarrow 0} r^{-d} \mu(B(p, 2 r) \backslash p \cdot \mathbb{V}(\delta s r))=0 .
$$

Hence we may assume that for some $r_{0}, 0<r_{0}<1 / 2$,

$$
\mu(B(p, 2 r) \backslash p \cdot \mathbb{V}(\delta s r))<b r^{d} \text { for } p \in A, 0<r \leq r_{0},
$$

with $b=2^{-1} \cdot C^{-d} \epsilon$. Finally, if $\mu(A)>0$, using the fact $\theta^{* d}(\mu\llcorner A, p)=$ $\theta^{* d}(\mu, p)$ for $\mu$-a.e. $p \in A$ (by Lemma 3.6), we may assume by (3.8) that for some $p_{0} \in A$ and with $r_{0}$ as above

$$
A \subset B\left(p_{0},\left(2^{4} \cdot C\right)^{-1} r_{0}\right) \text { with } \mu(A)>c r_{0}^{d} .
$$

We shall show that this leads to a contradiction.

Under the above assumptions we have that for all $p \in \mathbb{H}^{n}$ and $0<r \leq r_{0} / 2$,

$$
A \cap B(p, r) \subset q \cdot \mathbb{V}(s r) \text { for } q \in A \cap B(p, r) .
$$

whenever $\mu(B(p, r)) \geq 2 b r^{d}$.

To see this let $q \in A \cap B(p, r)$ and suppose there is $q^{\prime} \in A \cap B(p, r) \backslash q \cdot \mathbb{V}(s r)$. Then by Lemma 2.24

$$
B(p, r) \subset(B(q, 2 r) \backslash q \cdot \mathbb{V}(\delta s r)) \cup\left(B\left(q^{\prime}, 2 r\right) \backslash q^{\prime} \cdot \mathbb{V}(\delta s r)\right),
$$

whence by (3.9)

$$
\mu(B(p, r)) \leq \mu(B(q, 2 r) \backslash q \cdot \mathbb{V}(\delta s r))+\mu\left(B\left(q^{\prime}, 2 r\right) \backslash q^{\prime} \cdot \mathbb{V}(\delta s r)\right)<2 b r^{d}
$$

which is a contradiction and proves (3.11). 
We may assume that

$$
\mathbb{V}=\left\{(p, t) \in \mathbb{H}^{n}: p_{i}=0 \text { for } i>d \text { and } t=0\right\},
$$

identified as $\mathbb{R}^{d}$. Let $k_{0}$ be the integer defined by $C \cdot 2^{2-k_{0}} \leq r_{0}<C \cdot 2^{3-k_{0}}$ and

$$
Q_{0}=\left\{\left(p_{1}, \ldots, p_{d}\right) \in \mathbb{V}: 0 \leq p_{i}<2^{-k_{0}}\right\} .
$$

Let $Q_{j_{k_{0}}}=Q_{0}$ and let $Q_{j_{k_{0}}, \ldots, j_{k}}, k=k_{0}+1, k_{0}+2, \ldots, j_{i}=1, \ldots, 2^{d}$, be the cubes of side-length $2^{-k}$ constituting the standard dyadic decomposition of $Q_{0}$ in such a way that $Q_{j_{k_{0}}, \ldots, j_{k+1}} \subset Q_{j_{k_{0}}, \ldots, j_{k}}$. Let $P: \mathbb{H}^{n} \rightarrow \mathbb{V}$ be the natural projection and set

$$
S_{0}=P^{-1}\left(Q_{0}\right), S_{j_{k_{0}}, \ldots, j_{k}}=P^{-1}\left(Q_{j_{k_{0}}, \ldots, j_{k}}\right) .
$$

By translating $A$ we may assume that $A \subset S_{0}$ by (3.10) and the choice $r_{0}<C$. $2^{3-k_{0}}$. Let $\mathcal{I}$ be the set of those multi-indices $\left(j_{k_{0}}, \ldots, j_{k}\right)$ such that

$$
\mu\left(A \cap S_{j_{k_{0}}, \ldots, j_{k}}\right)<\epsilon 2^{-k d}=\epsilon \mathcal{L}^{d}\left(Q_{j_{k_{0}}, \ldots, j_{k}}\right)
$$

and

$$
\mu\left(A \cap S_{j_{k_{0}}, \ldots, j_{l}}\right) \geq \epsilon 2^{-l d}
$$

for all $l<k$, and let $\mathcal{J}$ be the set of those multi-indices $\left(j_{k_{0}}, \ldots, j_{k}\right)$ such that

$$
\mu\left(A \cap S_{j_{k_{0}}, \ldots, j_{l}}\right) \geq \epsilon 2^{-l d}
$$

for all $1 \leq l \leq k$.

Then the sets $S_{\iota}, \iota \in \mathcal{I}$, are disjoint. We shall prove that for each $\iota \in \mathcal{I} \cup \mathcal{J}, \iota=$ $\left(j_{k_{0}}, \ldots, j_{k}\right)$, there is $p_{\iota} \in A$ such that

$$
A \cap S_{\iota} \subset B\left(p_{\iota}, C \cdot 2^{-k}\right) .
$$

Then

$$
A \subset \bigcup_{\iota \in \mathcal{I}} S_{\iota}
$$

Otherwise there is $p \in A$ and $j_{k_{0}}, j_{k_{0}+1}, \ldots$, such that $\iota(k)=\left(j_{k_{0}}, \ldots, j_{k}\right) \in \mathcal{J}$ and $p \in A \cap S_{\iota(k)}$ for every $k$. As

$$
p \in A \cap S_{\iota(k)} \subset B\left(p_{\iota(k)}, C \cdot 2^{-k}\right) \subset B\left(p, C \cdot 2^{1-k}\right)
$$

we get a contradiction from (3.7) and (3.12). Hence

$$
\begin{aligned}
& \mu(A) \leq \sum_{\iota \in \mathcal{I}} \mu\left(A \cap S_{\iota}\right) \leq \epsilon \sum_{\iota \in \mathcal{I}} \mathcal{L}^{d}\left(Q_{\iota}\right) \leq \\
& \epsilon \mathcal{L}^{d}\left(Q_{0}\right)=\epsilon \cdot 2^{-k_{0} d}<\epsilon r_{0}^{d}<c r_{0}^{d},
\end{aligned}
$$

which is a contradiction with (3.10), gives us that $\mu(A)=0$ and proves the theorem. 
We prove (3.13) by induction on $k$ starting with $k=k_{0}$. This case is clear by (3.10). Suppose $k$ is such that (3.13) holds for all $\iota=\left(j_{1}, \ldots, j_{l}\right) \in \mathcal{I} \cup \mathcal{J}, l<k$. Let $\kappa=\left(j_{1}, \ldots, j_{k}\right) \in \mathcal{I} \cup \mathcal{J}$. Then $\iota=\left(j_{1}, \ldots, j_{k-1}\right) \in \mathcal{J}$ and (3.13) holds for $p_{\iota} \in A$. So

$$
A \cap S_{\kappa} \subset A \cap S_{\iota} \subset A \cap B\left(p_{\iota}, C \cdot 2^{1-k}\right) .
$$

Since $\iota \in \mathcal{J}$, we have, by (3.12), that

$$
\mu\left(A \cap B\left(p_{\iota}, C \cdot 2^{1-k}\right) \geq \mu\left(A \cap S_{\iota}\right) \geq \epsilon 2^{(1-k) d}=2 b\left(C \cdot 2^{1-k}\right)^{d},\right.
$$

and so we have by (3.11), since $C \cdot 2^{1-k} \leq r_{0} / 2$, that

$$
A \cap S_{\kappa} \subset p_{\iota} \cdot \mathbb{V}\left(s C \cdot 2^{1-k}\right) \cap S_{\iota}=p_{\iota} \cdot \mathbb{V}\left(2^{-k}\right) \cap S_{\iota} .
$$

As $Q_{\iota}$ is contained in a ball of radius $d \cdot 2^{-k}$, we have by Lemma 2.25

$$
\operatorname{diam}\left(p_{\iota} \cdot \mathbb{V}\left(2^{-k}\right) \cap S_{\iota}\right) \leq C \cdot 2^{-k}
$$

from which (3.13) follows for $\kappa$.

\subsection{Intrinsic rectifiable sets}

The notion of H-regular surfaces introduced in Definition 2.10 allows to give the following definition of intrinsic rectifiable sets. This definition was given in [14], for dimension $2 n$, to prove De Giorgi's structure result for sets of finite perimeter in Heisenberg groups, and later for any dimension in [17] and in Carnot groups of [15, Step 2].

Definition 3.11. We say that $E \subseteq \mathbb{H}^{n}$ is d-dimensional $\mathbb{H}$-rectifiable, or $E$ is $(d, \mathbb{H})$-rectifiable, if there exists a sequence of $d$-dimensional $\mathbb{H}$-regular surfaces $\left(S_{i}\right)_{i \in \mathbb{N}}$ such that

$$
\mathcal{S}^{d_{m}}\left(E \backslash \bigcup_{i \in \mathbb{N}} S_{i}\right)=0,
$$

where $d_{m}=d$ if $1 \leq d \leq n$ and $d_{m}=d+1$ if $n+1 \leq d \leq 2 n$.

Remark 3.12. We recall that, for $n+1 \leq d \leq 2 n$, a $d$-dimensional Euclidean rectifiable set $E \subset \mathbb{R}^{2 n+1} \equiv \mathbb{H}^{n}$ always is a $(d, \bar{H})$-rectifiable set, while the converse is false. On the contrary, for $1 \leq d \leq n$, a $(d, \mathbb{H})$-rectifiable set is $d$-dimensional Euclidean rectifiable while the opposite is false (see [7], [15] and [17]).

At the moment we are unable to prove our main theorem for $(d, \mathbb{H})$-rectifiable sets when $1 \leq d \leq n$. Hence we give an alternative definition. In Euclidean spaces these two definitions are equivalent and in fact the type of definition below was the original definition of Federer and it has also often been used in general metric spaces. We don't know if they are equivalent in $\mathbb{H}^{n}$. The problem is that we don't have a suitable Whitney extension theorem for maps $f: F \rightarrow \mathbb{H}^{n}$ when $F$ is a closed subset of $\mathbb{R}^{d}$. 
Definition 3.13. Let $1 \leq d \leq n$. We say that $E \subset \mathbb{H}^{n}$ is $\left(d, \mathbb{H}_{L}\right)$-rectifiable if there exist Lipschitz mappings $f_{i}: A_{i} \rightarrow \mathbb{H}^{n}, A_{i} \subset \mathbb{R}^{d}, i=1,2, \ldots$, such that

$$
\mathcal{S}^{d}\left(E \backslash \bigcup_{i \in \mathbb{N}} f_{i}\left(A_{i}\right)\right)=0 .
$$

Note that we can always take the sets $A_{i}$ to be compact since the Lipschitz maps $f_{i}$ trivially extend to the closures of the sets $A_{i}$ and closed sets are countable unions of compact sets.

The domain of definition for the Lipschitz maps in the definition of $\left(d, \mathbb{H}_{L}\right)$ rectifiability can be taken to be all of $\mathbb{R}^{d}$, because of the Lipschitz extension results proved in [6,22] and [34].

Notice also that $(d, \mathbb{H})$-rectifiability implies $\left(d, \mathbb{H}_{L}\right)$-rectifiability, for $1 \leq d \leq$ $n$. Indeed, a continuous Pansu differentiable function $\varphi: \mathcal{V} \subset \mathbb{R}^{d} \rightarrow \mathbb{H}^{n}$ is also locally Lipschitz (see [17, Theorem 3.5]).

\subsection{Main Theorem}

Now we can state the main results of this paper.

Theorem 3.14. Let $1 \leq d \leq n$ be an integer and $E \subset \mathbb{H}^{n}$ be a Borel set such that $\mathcal{S}^{d}\llcorner E$ is locally finite. Then the following conditions are equivalent:

(i) $E$ is $\left(d, \mathbb{H}_{L}\right)$-rectifiable.

(ii) For $\mathcal{S}^{d}$-a.e. $p \in E$ there is $\mathbb{T}_{p} \in \mathcal{G}\left(\mathbb{H}^{n}, d\right)$ with

$$
\frac{1}{r^{d}} T_{p, r \#}\left(\mathcal { S } ^ { d } \llcorner E ) \rightarrow \mathcal { S } ^ { d } \left\llcorner\mathbb{T}_{p}, \quad \text { as } r \rightarrow 0,\right.\right.
$$

weakly in the sense of measures.

(iii) For $\mathcal{S}^{d}$-a.e. $p \in E$ there is $\mathbb{T}_{p} \in \mathcal{G}\left(\mathbb{H}^{n}, d\right)$ such that

$$
\mathbb{T}_{p}=\operatorname{apTan}_{\mathbb{H}}^{d}(E, p) .
$$

(iv) For $\mathcal{S}^{d}$-a.e. $p \in E$ there is $\mathbb{T}_{p} \in \mathcal{G}\left(\mathbb{H}^{n}, d\right)$ such that

$$
\operatorname{Tan}\left(\mathcal{S}^{d}\llcorner E, p)=\left\{c \mathcal{S}^{d}\left\llcorner\mathbb{T}_{p}: 0<c<\infty\right\} .\right.\right.
$$

Finally, if $d=1$, (i) to (iv) are also equivalent with:

(v) for $\mathcal{S}^{1}$-a.e. $p \in E$ there exists a Radon measure $\lambda_{p}$ such that

$$
\operatorname{Tan}\left(\mathcal{S}^{1}\llcorner E, p)=\left\{c \lambda_{p}: 0<c<\infty\right\} .\right.
$$

Notice that (v) does not imply the other conditions if $d>1$. Indeed, for any $2 \leq$ $d \leq n$, take $E$ a $(d-1)$-dimensional vertical subgroup. Then $\operatorname{Tan}\left(\mathcal{S}^{d}\llcorner E, p)=\right.$ $\left\{c \overline{\mathcal{S}}^{d}{ }_{\llcorner} E, 0<c<\infty\right\}$ but $E \notin \mathcal{G}\left(\mathbb{H}^{n}, d-1\right)$. 
Theorem 3.15. Let $n+1 \leq d \leq 2 n$ be an integer and $E \subset \mathbb{H}^{n}$ be a Borel set such that $\mathcal{S}^{d_{m}}\llcorner E$ is locally finite. Then the following conditions are equivalent:

(i) $E$ is $(d, \mathbb{H})$-rectifiable.

(ii) For $\mathcal{S}^{d_{m}}$-a.e. $p \in E$ there is $\mathbb{T}_{p} \in \mathcal{G}\left(\mathbb{H}^{n}, d\right)$ with

$$
\frac{1}{r^{d_{m}}} T_{p, r \#}\left(\mathcal { S } ^ { d _ { m } } \llcorner E ) \rightarrow \mathcal { S } ^ { d _ { m } } \left\llcorner\mathbb{T}_{p}, \quad \text { as } r \rightarrow 0,\right.\right.
$$

weakly in the sense of measures.

(iii) For $\mathcal{S}^{d_{m}}$-a.e. $p \in E, \Theta_{*}^{d_{m}}(E, p)>0$ and there is $\mathbb{T}_{p} \in \mathcal{G}\left(\mathbb{H}^{n}, d\right)$ such that

$$
\mathbb{T}_{p}=\operatorname{apTan}_{\mathbb{H}}^{d}(E, p) .
$$

(iv) For $\mathcal{S}^{d_{m}}$-a.e. $p \in E, \Theta_{*}^{d_{m}}(E, p)>0$ and there is $\mathbb{T}_{p} \in \mathcal{G}\left(\mathbb{H}^{n}, d\right)$ such that

$$
\operatorname{Tan}\left(\mathcal{S}^{d_{m}}\llcorner E, p)=\left\{c \mathcal{S}^{d_{m}}\left\llcorner\mathbb{T}_{p}: 0<c<\infty\right\} .\right.\right.
$$

(v) For $\mathcal{S}^{d_{m}}$-a.e. $p \in E, \Theta_{*}^{d_{m}}(E, p)>0$ and there exists a Radon measure $\lambda_{p}$ such that

$$
\operatorname{Tan}\left(\mathcal{S}^{d_{m}}\llcorner E, p)=\left\{c \lambda_{p}: 0<c<\infty\right\} .\right.
$$

Before proving the two main theorems we state the following corollary that gives the Heisenberg version of a different easier characterization of rectifiable sets (see for instance [1, Theorem 2.61]).

Corollary 3.16. Let $d$ be an integer with $1 \leq d \leq 2 n$ and $d_{m}$ as in (2.13). Let $E \subset \mathbb{H}^{n}$ be a Borel set with $\mathcal{S}^{d_{m}}(E)<\infty$. Assume that for all $p \in E$ there are $r_{p}>0, c_{p}>0$ and $\mathbb{T}_{p} \in \mathcal{G}\left(\mathbb{H}^{n}, d\right)$ such that

$$
E \cap B\left(p, r_{p}\right) \subset X\left(p, \mathbb{T}_{p}, c_{p}\right) .
$$

Then

(i) if $1 \leq d \leq n, E$ is $\left(d, \mathbb{H}_{L}\right)$-rectifiable

(ii) if $n+1 \leq d \leq 2 n$ and $\Theta_{*}^{d_{m}}(E, p)>0$ for $\mathcal{S}^{d_{m}}$-a.e. $p \in E$, $E$ is $(d, \mathbb{H})$ rectifiable.

Proof of Theorems 3.14 and 3.15 . We shall prove both theorems simultaneously making only the different arguments in appropriate places. The scheme of the implications in the proof is the following: (i) $\Rightarrow$ (ii) $\Rightarrow$ (iv) $\Rightarrow$ (iii) $\Rightarrow$ (i), and (iv) $\Longleftrightarrow$ (v).

1. Proof of (i) $\Rightarrow$ (ii) We first prove the case $n+1 \leq d \leq 2 n$. By assumption

$$
E=E_{0} \cup\left(\cup_{i=1}^{\infty} E_{i}\right)
$$


where $\mathcal{S}^{d_{m}}\left(E_{0}\right)=0$ and, for $i \geq 1, E_{i} \subset S_{i}$ with $S_{i}$ is a $d$-dimensional $\mathbb{H}$-regular surface. By (3.1) for a fixed $i \geq 1$, at each $p \in S_{i}$,

$$
\frac{1}{r^{d_{m}}} T_{p, r \#}\left(\mathcal { S } ^ { d _ { m } } \llcorner S _ { i } ) \rightarrow \mathcal { S } ^ { d _ { m } } \left\llcornerT_{\mathbb{H}} S_{i}(p) \text { as } r \rightarrow 0 .\right.\right.
$$

On the other hand, by Lemma 3.6, for a fixed $i$ and for $\mathcal{S}^{d_{m}}$-a.e. $p \in E_{i}$,

$$
\Theta^{* d_{m}}\left(E \backslash E_{i}, p\right)=0 \quad \text { and } \quad \Theta^{* d_{m}}\left(S_{i} \backslash E_{i}, p\right)=0 .
$$

Now observe that for $p \in E_{i}$ and for $\varphi \in C_{c}^{0}\left(\mathbb{H}^{n}\right)$,

$$
\begin{aligned}
& \frac{1}{r^{d_{m}}} \int_{E} \varphi \circ T_{p, r} d \mathcal{S}^{d_{m}}= \\
& =\frac{1}{r^{d_{m}}} \int_{E \backslash E_{i}} \varphi \circ T_{p, r} d \mathcal{S}^{d_{m}}+\frac{1}{r^{d_{m}}} \int_{S_{i}} \varphi \circ T_{p, r} d \mathcal{S}^{d_{m}}- \\
& \frac{1}{r^{d_{m}}} \int_{S_{i} \backslash E_{i}} \varphi \circ T_{p, r} d \mathcal{S}^{d_{m}} .
\end{aligned}
$$

Thus by (3.14) and (3.15) taking the limit as $r \rightarrow 0$ in (3.16) we get that, for $\mathcal{S}^{d_{m}}$-a.e. $p \in E$, (ii) holds with $\mathbb{T}_{p} \equiv T_{\mathbb{H}} S_{i}(p) \in \mathcal{G}\left(\mathbb{H}^{n}, d\right)$.

Now we consider the case $1 \leq d \leq n$. Since $E$ is $\left(d, \mathbb{H}_{L}\right)$-rectifiable, there are compact sets $C_{i} \subset \mathbb{R}^{d}$ and Lipschitz maps $f_{i}: C_{i} \rightarrow \mathbb{H}^{n}$ such that

$$
\mathcal{S}^{d}\left(E \backslash \bigcup_{i \in \mathbb{N}} f_{i}\left(C_{i}\right)\right)=0 .
$$

Using Theorem 2.6 and Propositions 4.3.3 and 4.3.1 in [21] we can further decompose the sets $C_{i}$ into null-sets and countable unions to obtain Borel sets $B_{i} \subset \mathbb{R}^{d}$ and Lipschitz maps $f_{i}: B_{i} \rightarrow \mathbb{H}^{n}$ with the following properties:

(i) $E=E_{0} \cup \bigcup_{i \in \mathbb{N}} S_{i}$ with $S_{i}=f_{i}\left(B_{i}\right)$ and $\mathcal{S}^{d}\left(E_{0}\right)=0$,

(ii) the sets $S_{i}$ are disjoint,

(iii) every $x \in B_{i}$ is a Lebesgue point of $J_{f_{i}} \chi_{B_{i}}$,

(iv) $f_{i}$ is Pansu differentiable and $\left(d_{H} f_{i}\right)_{x}$ is injective at every $x \in B_{i}$,

(v) $f_{i}$ is bi-Lipschitz.

The Jacobian for a map $f$ at a point $x$ of differentiability is defined by

$$
J_{f}(x)=\frac{\mathcal{S}^{d}\left(d_{H} f_{x}\left(B_{d, \mathrm{euc}}(0,1)\right)\right)}{\mathcal{L}^{d}\left(B_{d, \mathrm{euc}}(0,1)\right)},
$$

where $B_{d, \text { euc }}$ is the Euclidean ball in $\mathbb{R}^{d}$. This is as in [21, Definition 4.2.1], except that we are using spherical Hausdorff measures instead of ordinary Hausdorff measures. It is easy to check from [21] that the area formula which we shall use with $J_{f}$ is also valid for the spherical Hausdorff measure. 
We shall now verify that (3.14) holds at an arbitrary point $p=f_{i}(x) \in S_{i}$, $x \in B_{i}$, with $T_{\mathbb{H}} S_{i}(p)$ replaced by $\left(d_{H} f_{i}\right)_{x}\left(\mathbb{R}^{d}\right)$. This will be enough since the rest of the argument goes as in the case $n+1 \leq d \leq 2 n$. For simplicity write $f=f_{i}, B=B_{i}$ and $S=S_{i}$. Let $\varphi \in C_{c}^{0}\left(\mathbb{H}^{n}\right)$. As $d_{H} f_{x}$ is injective and $f$ is biLipschitz, it follows that there is a positive number $c$ such that $\left\|d_{H} f_{x}(y)\right\| \geq c|y|_{\mathbb{R}^{d}}$ for $y \in \mathbb{R}^{d}$ and $d(f(x), f(y)) \geq c|x-y|_{\mathbb{R}^{d}}$ for $y \in B$. Let $R>0$ be such that $\operatorname{spt} \varphi \subset B(0, R)$. Then

$$
\varphi\left(\delta_{1 / r}\left(p^{-1} \cdot f(y)\right)\right)=\varphi\left(d_{H} f_{x}((y-x) / r)\right)=0
$$

if $|x-y|_{\mathbb{R}^{d}} \geq \operatorname{Rr} / c$. Using this, the definition of $d_{H} f_{x}$ and the fact that $x$ is a Lebesgue point of $J_{f} \chi_{B}$, one checks easily that

$$
\left.\int_{B} \varphi\left(\delta_{1 / r}\left(p^{-1} \cdot f(y)\right)\right) J_{f}(y) d y-J_{f}(x) \int_{B} \varphi\left(d_{H} f_{x}((y-x) / r)\right)\right) d y=o\left(r^{d}\right)
$$

as $r \rightarrow 0$.

By a simple change of variable (note that $\mathcal{S}^{d}\left\llcorner d_{H} f_{x}\left(\mathbb{R}^{d}\right)\right.$ is just the Lebesgue measure on the $d$-plane $d_{H} f_{x}\left(\mathbb{R}^{d}\right)$ ) we have, for any $r>0$,

$$
\begin{aligned}
\int_{\mathbb{H}^{n}} \varphi d\left(\mathcal{S}^{d}\left\llcorner d_{H} f_{x}\left(\mathbb{R}^{d}\right)\right)\right. & =\int_{d f_{x}\left(\mathbb{R}^{d}\right)} \varphi d \mathcal{S}^{d} \\
& \left.=r^{-d} J_{f}(x) \int_{\mathbb{R}^{d}} \varphi\left(d_{H} f_{x}((y-x) / r)\right)\right) d y .
\end{aligned}
$$

By the area formula, see [21, Corollary 4.3.6],

$$
\begin{aligned}
\int_{B} \varphi\left(\delta_{1 / r}\left(p^{-1} \cdot f(y)\right)\right) J_{f}(y) d y & =\int_{S} \varphi\left(\delta_{1 / r}\left(p^{-1} \cdot q\right)\right) d \mathcal{S}^{d}(q) \\
& =\int_{\mathbb{H}^{n}} \varphi d\left(T_{p, r \#}\left(\mathcal{S}^{d}\llcorner S)\right) .\right.
\end{aligned}
$$

Using these we get

$$
\begin{aligned}
\int_{\mathbb{H}^{n}} \varphi d\left(\mathcal{S}^{d}\left\llcorner d_{H} f_{x}\left(\mathbb{R}^{d}\right)\right)\right. & \left.=\lim _{r \rightarrow 0} r^{-d} J_{f}(x) \int \varphi\left(d_{H} f_{x}((y-x) / r)\right)\right) d y \\
& =\lim _{r \rightarrow 0} \frac{1}{r^{d}} \int_{B} \varphi\left(\delta_{1 / r}\left(p^{-1} \cdot f(y)\right)\right) J_{f}(y) d y \\
& =\lim _{r \rightarrow 0} \frac{1}{r^{d}} \int \varphi d\left(T_{p, r \#}\left(\mathcal{S}^{d}\llcorner S)\right) .\right.
\end{aligned}
$$

This completes the proof of the implication (i) $\Rightarrow$ (ii). 
2. Proof of (ii) $\Rightarrow$ (iv)

By definition, $v \in \operatorname{Tan}\left(\mathcal{S}^{d_{m}}\llcorner E, p)\right.$ if there are positive sequences $\left(c_{i}\right)_{i}$ and $\left(r_{i}\right)_{i}$ with $\lim _{i} r_{i}=0$ such that

$$
c_{i} T_{p, r_{i} \#}\left(\mathcal{S}^{d_{m}}\llcorner E) \rightarrow v, \quad \text { as } i \rightarrow+\infty .\right.
$$

On the other hand, by assumption (ii), we know that

$$
T_{p, r \#}\left(\mathcal{S}^{d_{m}}\llcorner E) / r^{d_{m}} \rightarrow \mathcal{S}^{d_{m}}\left\llcorner\mathbb{T}_{p}, \quad \text { as } r \rightarrow 0 .\right.\right.
$$

Hence $\lim _{i} r_{i}^{d_{m}} c_{i}$ exists and

$$
c_{i} T_{p, r_{i} \#}\left(\mathcal { S } ^ { d _ { m } } \llcorner E ) \rightarrow ( \operatorname { l i m } _ { i \rightarrow + \infty } r _ { i } ^ { d _ { m } } c _ { i } ) \mathcal { S } ^ { d _ { m } } \left\llcorner\mathbb{T}_{p}\right.\right.
$$

Moreover, (ii) implies easily that $\Theta^{d_{m}}(E, p)=1$ for $\mathcal{S}^{d_{m}}$-a.e. $p \in E$.

3. Proof of (iv) $\Rightarrow$ (iii)

By our assumption and Theorem 3.3 we have for $\mathcal{S}^{d_{m}}$-a.e. $p \in E$ that for some positive number $c$,

$$
\frac{1}{\mathcal{S} d_{m}(E \cap(B(p, r))} T_{p, r \#}\left(\mathcal { S } ^ { d _ { m } } \llcorner E ) \rightarrow c \mathcal { S } ^ { d _ { m } } \left\llcorner\mathbb{T}_{p}, \quad \text { as } r \rightarrow 0 .\right.\right.
$$

Since $\mathcal{S}^{d_{m}}\left(\mathbb{T}_{p} \cap \partial\left(B(e, 1) \backslash X\left(e, \mathbb{T}_{p}, s\right)\right)\right)=0$ for every $s \in(0,1)$, we infer

$$
\begin{aligned}
& \lim _{r \rightarrow 0} \frac{\mathcal{S}^{d_{m}}\left\llcorner E\left(B(p, r) \backslash X\left(p, \mathbb{T}_{p}, s\right)\right)\right.}{\mathcal{S}^{d_{m}}(E \cap(B(p, r))} \\
& =\lim _{r \rightarrow 0} \frac{1}{\mathcal{S}^{d_{m}}(E \cap(B(p, r))} T_{p, r \#}\left(\mathcal{S}^{d_{m}}\llcorner E)\left(B(e, 1) \backslash X\left(e, \mathbb{T}_{p}, s\right)\right)\right. \\
& =\mathcal{S}^{d_{m}}\left\llcorner\mathbb{T}_{p}\left(B(e, 1) \backslash X\left(e, \mathbb{T}_{p}, s\right)\right)=0 .\right.
\end{aligned}
$$

Since by Lemma 3.6, $\Theta^{* d_{m}}(E, p)>0$ for $\mathcal{S}^{d_{m}}$-a.e. $p \in E$, we obtain that $\mathbb{T}_{p}$ is an approximate tangent subgroup. Almost everywhere uniqueness of the approximate tangent subgroup follows from Proposition 3.9.

4. Proof of (iii) $\Longrightarrow$ (i)

Because $d$-dimensional $\mathbb{H}$-regular surfaces are defined differently if $d$ is smaller or larger than $n$, we have to divide the proof of the implication (iii) $\Longrightarrow$ (i) in two parts.

Assume $n+1 \leq d \leq 2 n$. By assumption, for $\mathcal{S}^{d_{m}}$-a.e. $p \in E$ there are $r(p)$, $l(p)>0$ and $\mathbb{T}_{p}=\overline{\operatorname{apTan}}_{\mathbb{H}}^{d}(E, p) \in \mathcal{G}\left(\mathbb{H}^{n}, d\right)$ such that, for $0<r<r(p)$,

$$
\mathcal{S}^{d_{m}}(E \cap B(p, r))>l(p) r^{d_{m}},
$$


and for all $0<s<1$,

$$
\lim _{r \rightarrow 0} \frac{\mathcal{S}^{d_{m}}\left\llcorner E\left(B(p, r) \backslash X\left(p, \mathbb{T}_{p}, s\right)\right)\right.}{r^{d_{m}}}=0 .
$$

For $i=1,2, \ldots$, let $E_{i}$ be the set of those $p \in E$ as above for which $r(p)>1 / i$ and $l(p)>1 / i$. Then $\mathcal{S}^{d_{m}}\left(E \backslash E^{*}\right)=0$ where $E^{*}=\cup_{i=1}^{\infty} E_{i}$.

Recall now that, from Lemma 2.32, we have that for all $p \in E^{*}$, there are $2 n+1-d$ horizontal unit vectors $\tilde{v}_{h}(p)=v_{h}\left(\mathbb{T}_{p}\right) \in \mathrm{HH}_{p}^{n}$, transversal to $\mathbb{T}_{p}$, such that

$$
\mathbb{V}_{p}:=\exp \left(\operatorname{span}\left\{\tilde{v}_{1}(p), \cdots, \tilde{v}_{2 n+1-d}(p)\right\}\right)
$$

is a horizontal subgroup of $\mathbb{H}^{n}$ and $\mathbb{H}^{n}$ is the semi-direct product of $\mathbb{V}_{p}$ and $\mathbb{T}_{p}=$ $\operatorname{apTan}_{\mathbb{H}}^{d}(E, p)$. Moreover, $E^{*}$ can be written as a countable union of $\mathcal{S}^{d_{m}}$ measurable sets $F_{j}$ such each $\tilde{v}_{h \mid F_{j}}$ is $\mathcal{S}^{d_{m}}$ measurable. We get this using the continuity part of Lemma 2.32 and Proposition 3.9. We see then that the functions $\tilde{v}_{h}: E^{*} \rightarrow H \mathbb{H}^{n}$ are measurable sections of $\mathrm{HH}^{n}$, for each $1 \leq h \leq 2 n+1-d$.

Define for $i, j=1,2 \ldots, h=1, \ldots, 2 n+1-d$, and $p \in E^{*}$,

$$
\rho_{i, h, j}(p)=\sup \left\{\frac{\left|\left\langle\tilde{v}_{h}(p), \pi\left(p^{-1} \cdot q\right)\right\rangle\right|}{d(p, q)}: q \in E_{i}, 0<d(p, q)<1 / j\right\} .
$$

We want to prove that for $i=1,2 \ldots, h=1, \ldots, 2 n+1-d$, and $p \in E^{*}$,

$$
\lim _{j \rightarrow \infty} \rho_{i, h, j}(p)=0
$$

Assume, by contradiction, that this fails. Then there is $s>0$ and for all $0<\tau<$ $1 / i$ there is $q \in E_{i}$ such that

$$
\left|\left\langle\tilde{v}_{h}(p), \pi\left(p^{-1} \cdot q\right)\right\rangle\right|>s d(p, q) \quad \text { and } 0<d(p, q)<\tau .
$$

Let $r=d(p, q)$. Then, by (3.20), $q \in B(p, r) \backslash X\left(p, \mathbb{N}\left(\tilde{v}_{h}(p)\right), s\right)$. Hence, from Lemma 2.29, we have

$$
B(q, s r / 2) \subset B(p, 2 r) \backslash X\left(p, \mathbb{N}\left(\tilde{v}_{h}(p)\right), s / 3\right) .
$$

By (2.26), (3.21) and (3.17),

$$
\begin{aligned}
& \mathcal{S}^{d_{m}}\left(E \cap B(p, 2 r) \backslash X\left(p, \mathbb{T}_{p}, s / 3\right)\right) \geq \\
& \mathcal{S}^{d_{m}}\left(E \cap B(p, 2 r) \backslash X\left(p, \mathbb{N}\left(\tilde{v}_{h}(p)\right), s / 3\right)\right) \geq \\
& \geq \mathcal{S}^{d_{m}}(E \cap B(q, s r / 2)) \geq(1 / i)(s r / 2)^{d_{m}} .
\end{aligned}
$$

This is a contradiction with (3.18) and proves (3.19).

Applying Lusin's theorem to each $\tilde{v}_{h}$ and Egoroff's theorem to the sequences $\left(\rho_{i, h, j}\right)_{j}$ we can write $E_{i}=E_{i, 0} \cup_{k=1}^{\infty} K_{i, k}$ such that $\mathcal{S}^{d_{m}}\left(E_{i, 0}\right)=0$, the sets $K_{i, k}$ 
are compact, each $\tilde{v}_{h \mid K_{i, k}}$ is continuous and $\rho_{i, h, j} \rightarrow 0$ as $j \rightarrow \infty$ uniformly on $K_{i, k}$. We can apply Whitney's Extension Theorem (Theorem 2.9) with $K \equiv K_{i, k}$, $f \equiv 0, k \equiv \tilde{v}_{h}$ getting the existence of a $C_{\mathbb{H}}^{1}$ function $\tilde{f}_{i, k, h}: \mathbb{H}^{n} \rightarrow \mathbb{R}$ with $\left(\tilde{f}_{i, k, h}\right)_{\mid K_{i, k}}=0, \nabla_{H} \tilde{f}_{i, k, h}=\tilde{v}_{h}$ and $\left|\nabla_{H} \tilde{f}_{i, k, h}\right| \neq 0$ on $K_{i, k}$.

Define $S_{i, k, h}:=\left\{p \in \mathbb{H}^{n}: \tilde{f}_{i, k, h}(p)=0,\left|\nabla_{H} \tilde{f}_{i, k, h}(p)\right|_{p} \neq 0\right\}$. Then $S_{i, k, h}$ is a 1 -codimensional $\mathbb{H}$-regular surface and $K_{i, k} \subset S_{i, k, h}$. Finally define

$$
S_{i, k}=\bigcap_{h=1}^{2 n+1-d} S_{i, k, h} .
$$

Recalling Remark 2.12 we have that $S_{i, k}$ is a $d$-dimensional $\mathbb{H}$-regular surface and $K_{i, k} \subset S_{i, k}(i=1,2, \ldots)$. Moreover

$$
E \subset E_{0} \cup\left(\cup_{i=1}^{\infty} \cup_{k=1}^{\infty} S_{i, k}\right)
$$

with $E_{0}=\left(E \backslash E^{*}\right) \cup \cup_{i=1}^{\infty} E_{i, 0}$ and $\mathcal{S}^{d_{m}}\left(E_{0}\right)=0$.

Now we shall deal with the case $1 \leq d \leq n$.

Let $s$ and $\eta$ be as in Lemma 2.23. Since, by Theorem 3.10, $\Theta_{*}^{d}(E, p)>0$ and $E$ has an approximate tangent subgroup $\mathbb{T}_{p}$ for $\mathcal{S}^{d}$-a.e. $p \in E$, we can write $E$ as the union of sets $E_{i}, i=0,1,2, \ldots$, with $\mathcal{S}^{d}\left(E_{0}\right)=0$ and such that for $i=$ $1,2, \ldots$, with some positive numbers $c_{i}$ and $r_{i}$, we have for $p \in E_{i}, 0<r<r_{i}$,

$$
\mathcal{S}^{d}(E \cap B(p, r)) \geq c_{i} r^{d}
$$

and

$$
\mathcal{S}^{d}\left(E \cap B(p, r) \backslash X\left(p, \mathbb{T}_{p}, s / 9\right)\right)<\epsilon_{i} r^{d}
$$

with $\epsilon_{i}=c_{i}(s / 4)^{d}$. Fix $i$ and let $\mathbb{T}_{1}, \ldots, \mathbb{T}_{k}$ be the subgroups given by Lemma 2.30 and corresponding to $s / 9$ in place of $s$. For every $p \in E_{i}$ there is $T_{j}=: \mathbb{T}_{i, j}$ with

$$
X\left(p, \mathbb{T}_{p}, s / 9\right) \subset X\left(p, \mathbb{T}_{i, j}, s / 3\right) .
$$

Hence we can decompose $E_{i}$ as a countable union of Borel sets $E_{i, j}$ such that $\operatorname{diam}\left(E_{i, j}\right)<r_{i} / 2$ and (3.23) holds for $p \in E_{i, j}$. Then for $p \in E_{i, j}, 0<r<r_{i}$,

$$
\mathcal{S}^{d}\left(E \cap B(p, r) \backslash X\left(p, \mathbb{T}_{i, j}, s / 3\right)\right)<\epsilon_{i} r^{d} .
$$

We check now that

$$
E_{i, j} \subset X\left(p, \mathbb{T}_{i, j}, s\right) \text { for } p \in E_{i, j} .
$$

Suppose there were $p, q \in E_{i, j}$ with $q \notin X\left(p, \mathbb{T}_{i, j}, s\right)$. Then $r=d(p, q) \leq$ $\operatorname{diam}\left(E_{i, j}\right)<r_{i} / 2$. By Lemma 2.29, $B(q, s r / 2) \subset B(p, 2 r) \backslash X\left(p, \mathbb{T}_{i, j}, s / 3\right)$. Hence by (3.22),

$$
\begin{aligned}
& \mathcal{S}^{d}\left(E \cap B(p, 2 r) \backslash X\left(p, \mathbb{T}_{i, j}, s / 3\right)\right) \geq \\
& \mathcal{S}^{d}(E \cap B(q, s r / 2)) \geq c_{i}(s r / 2)^{d}=\epsilon_{i}(2 r)^{d} .
\end{aligned}
$$


This contradicts with (3.24) and proves (3.25), which implies that $d\left(p^{-1} q, \mathbb{T}_{i, j}\right)<$ $s d(p, q)$ for $p, q \in E_{i, j}$ and $p \neq q$. Hence, by Lemma 2.23, the projection $\mathbb{H}^{n} \rightarrow \mathbb{T}_{i, j}$, restricted to $E_{i, j}$, is $1-1$ with Lipschitz inverse. As $\mathbb{T}_{i, j}$ is isometric with $\mathbb{R}^{d}$, the rectifiability of $E$ follows from this.

5. Proof of (iv) $\Rightarrow$ (v)

It is immediate by definition.

6. Proof of $(v) \Rightarrow(i v)$

From $(v)$ and Theorem 3.3 we get for $\mathcal{S}^{d_{m}}$-a.e. $p \in E$ the existence of a homogeneous subgroup $\mathbb{T}_{p}$ of $\mathbb{H}^{n}$ such that $\lambda_{p}$ is a left Haar measure of $\mathbb{T}_{p}$ and by Proposition 2.20 we have with $\alpha=\operatorname{dim}_{\mathbb{H}} \mathbb{T}_{p}$ that

$$
\lambda_{p}=c \mathcal{S}^{\alpha}\left\llcorner\mathbb{T}_{p}\right.
$$

So for $q \in \mathbb{T}_{p}$ and $r>0$,

$$
\lambda_{p}(B(q, r))=\lambda_{p}(B(e, 1)) r^{\alpha} .
$$

For $\mathcal{S}^{d_{m}}$-a.e. $p \in E$ we have, by (i) of Lemma 3.6, that

$$
2^{-d_{m}} \leq \Theta^{* d_{m}}(E, p) \leq 1
$$

Hence for all sufficiently small $r>0$,

$$
\mathcal{S}^{d_{m}}\left(E \cap(B(p, r)) \leq 2 \alpha_{d_{m}} r^{d_{m}},\right.
$$

and for some sequence $r_{i}>0, r_{i} \rightarrow 0$,

$$
\mathcal{S}^{d_{m}}\left(E \cap\left(B\left(p, r_{i}\right)\right) \geq 2^{-d_{m}-1} \alpha_{d_{m}} r_{i}^{d_{m}}\right.
$$

Since $\lambda_{p}(\partial B(e, \rho))=0$ for all $\rho>0$, we get thus by (3.26) and Theorem 3.3 that

$$
\lambda_{p}(B(e, 1)) \rho^{\alpha}=\lim _{i \rightarrow \infty} \frac{\mathcal{S}^{d_{m}}\left(E \cap\left(B\left(p, \rho r_{i}\right)\right)\right.}{\mathcal{S}^{d_{m}}\left(E \cap\left(B\left(p, r_{i}\right)\right)\right.} \leq 2^{d_{m}+2} \rho^{d_{m}} .
$$

Since this holds for all $\rho>0$, we must have $\alpha=d_{m}$, whence $\lambda_{p}=c \mathcal{S}^{d_{m}}\left\llcorner\mathbb{T}_{p}\right.$, and if $d=1$ or $d>n$ then $\mathbb{T}_{p} \in \mathcal{G}\left(\mathbb{H}^{n}, d\right)$.

\section{References}

[1] L. Ambrosio, N. Fusco and D. Pallara, "Functions of Bounded Variation and Free Discontinuity Problems", Oxford Mathematical Monographs, Oxford University Press, 2000.

[2] L. Ambrosio and B. KirChHeIM, Rectifiable sets in metric and Banach spaces, Math. Ann. 318 (2000), 527-555. 
[3] L. Ambrosio, B. Kleiner and E. Le Donne, Rectifiability of sets of finite perimeter in Carnot groups: existence of a tangent hyperplane, J. Geom. Anal. 19 (2009), 509-540.

[4] L. Ambrosio, F. Serra Cassano and D. Vittone, Intrinsic regular hypersurfaces in Heisenberg groups, J. Geom. Anal. 16 (2006), 187-232.

[5] G. ARENA and R. SERAPIONI, Intrinsic regular submanifolds in Heisenberg groups are differentiable graphs, Calc. Var. Partial Differential Equations, 35 (2009), 17-49.

[6] Z. BALOGH and K. S. FÄSSLER, Rectifiability and Lipschitz extensions into the Heisenberg group, Math. Z. 263 (2009), 673-683.

[7] Z. BAlogh, M. Rickly and F. Serra Cassano, Comparison of Hausdorff measures with respect to the Heisenberg metric, Publ. Mat. 47 (2003), 237-259.

[8] A. Bonfiglioli, E. LAnConelli and F. Uguzzoni, "Stratified Lie Groups and Potential Theory for their Sub-Laplacians", Springer Monographs in Mathematics, Springer-Verlag, 2007.

[9] L. Capogna, D. Danielli, S. D. Pauls and J. T. Tyson, "An Introduction to the Heisenberg Group and the sub-Riemannian Isoperimetric Problem", Birkhäuser, 2007.

[10] J. CHEEGER and B. KLEINER, Differentiating maps into $L^{1}$ and the geometry of $B V$ functions, Ann. of Math. 171 (2010), 1347-1385.

[11] G. CitTi and M. MANFredini, Implicit function theorem in Carnot Carathéodory spaces, Commun. Contemp. Math. 8, (2006), 253-293.

[12] H. Federer, "Geometric Measure Theory", Springer-Verlag, 1969.

[13] G. B. Folland and E. M. Stein, "Hardy spaces in Homogeneous Groups", Princeton University Press, 1982.

[14] B. Franchi, R. Serapioni and F. Serra Cassano, Rectifiability and perimeter in the Heisenberg group, Math. Ann. 321 (2001), 479-531.

[15] B. Franchi, R. Serapioni and F. SERRA CASSANo, On the structure of finite perimeter sets in step 2 Carnot groups, J. Geom. Anal. 13 (2003), 421-466.

[16] B. FrAnChI, R. SERAPIONI and F. SERRA CASSANO, Regular hypersurfaces, intrinsic perimeter and implicit function theorem, Comm. Anal. Geom. 11 (2003), 909-944.

[17] B. Franchi, R. SERAPIONI and F. SERRA CASSANO, Regular submanifolds, graphs and area formula in Heisenberg Groups, Adv. Math. 211 (2007), 152-203.

[18] B. Franchi, R. Serapioni and F. Serra Cassano, Differentiability of intrinsic Lipschitz functions within Heisenberg groups, J. Geom. Anal. DOI 10:1007/s12220-010-9178.

[19] M. Gromov, Carnot-Carathéodory spaces seen from within, In: "Subriemannian Geometry", Progress in Mathematics, Vol. 144, A. Bellaiche and J. Risler (eds.), Birkhäuser Verlag, Basel, 1996.

[20] B. Kirchheim and F. Serra Cassano, Rectifiability and parameterization of intrinsic regular surfaces in the Heisenberg group, Ann. Scuola Norm. Sup. Pisa Cl. Sci. (5) 3 (2004), 871-896.

[21] V. Magnani, "Elements of Geometric Measure Theory on Sub-Riemannion groups", Edizioni della Normale, Pisa, 2002.

[22] V. Magnani, Contact equations, Lipschitz extensions and isoperimetric inequalities, preprint http://cvgmt.sns.it/cgi/get.cgi/papers/maga/ (2009).

[23] P. Mattila, "Geometry of Sets and Measures in Euclidean Spaces", Cambridge University Press, 1995.

[24] P. Mattila, Measures with unique tangent measures in metric groups, Math. Scand. 97 (2005), 298-308.

[25] J. Mitchell, On Carnot-Carathèodory metrics, J. Differential Geom. 21 (1985), 35-45.

[26] R. Montgomery, "A Tour of Sub-Riemannian Geometries, their Geodesics and Applications", Mathematical Survey and Monographs, Vol. 91, American Mathematical Society, Providence RI, 2002.

[27] P. Pansu, Métriques de Carnot-Carathéodory et quasiisométries des espaces symétriques de rang un, Ann. of Math. 129 (1989), 1-60. 
[28] S. D. PAULS, A notion of rectifiability modelled on Carnot groups, Indiana Univ. Math. J. 53 (2004), 49-81.

[29] D. PREISS, Geometry of Measures in $\mathbb{R}^{n}$ : distribution, rectifiability and densities, Ann. of Math. 125 (1987), 537-643.

[30] S. RIGOT, Counter example to the isodiametric inequality in H-type groups, preprint (2004).

[31] L. Simon, "Lectures on Geometric Measure Theory", Proceedings of the Centre for Mathematical Analysis, Australian National University, Vol. 3, 1983.

[32] E. M. Stein, "Harmonic Analysis: Real Variable Methods, Orthogonality and Oscillatory Integrals", Princeton University Press, Princeton, 1993.

[33] N. Th. VAropoulos, L. SalofF-Coste and T. Coulhon, "Analysis and Geometry on Groups", Cambridge University Press, Cambridge, 1992.

[34] S. Wenger and R. Young, Lipschitz extensions into jet space Carnot group, Math. Res. Lett., to appear.

Department of Mathematics and Statistics

University of Helsinki

FI-00014, Finland

pertti.mattila@helsinki.fi

Dipartimento di Matematica Università di Trento

Via Sommarive, 14

38050 Povo (Trento), Italia serapion@science.unitn.it cassano@science.unitn.it 